\title{
Nonlinear Forced Vibration of a Viscoelastic Buckled Beam with 2 : 1 Internal Resonance
}

\author{
Liu-Yang Xiong, ${ }^{1}$ Guo-Ce Zhang, ${ }^{1}$ Hu Ding, ${ }^{1}$ and Li-Qun Chen ${ }^{1,2}$ \\ ${ }^{1}$ Shanghai Key Laboratory of Mechanics in Energy Engineering, Shanghai Institute of Applied Mathematics and Mechanics, \\ Shanghai University, 149 Yan Chang Road, Shanghai 200072, China \\ ${ }^{2}$ Department of Mechanics, Shanghai University, Shanghai 200444, China
}

Correspondence should be addressed to Hu Ding; dinghu3@shu.edu.cn

Received 30 October 2013; Revised 1 December 2013; Accepted 2 December 2013; Published 8 January 2014

Academic Editor: Sergio Preidikman

Copyright (C) 2014 Liu-Yang Xiong et al. This is an open access article distributed under the Creative Commons Attribution License, which permits unrestricted use, distribution, and reproduction in any medium, provided the original work is properly cited.

\begin{abstract}
Nonlinear dynamics of a viscoelastic buckled beam subjected to primary resonance in the presence of internal resonance is investigated for the first time. For appropriate choice of system parameters, the natural frequency of the second mode is approximately twice that of the first providing the condition for $2: 1$ internal resonance. The ordinary differential equations of the two mode shapes are established using the Galerkin method. The problem is replaced by two coupled second-order differential equations with quadratic and cubic nonlinearities. The multiple scales method is applied to derive the modulation-phase equations. Steady-state solutions of the system as well as their stability are examined. The frequency-amplitude curves exhibit the steadystate response in the directly excited and indirectly excited modes due to modal interaction. The double-jump, the saturation phenomenon, and the nonperiodic region phenomena are observed illustrating the influence of internal resonance. The validity range of the analytical approximations is assessed by comparing the analytical approximate results with a numerical solution by the Runge-Kutta method. The unstable regions in the internal resonance are explored via numerical simulations.
\end{abstract}

\section{Introduction}

The main goal of this paper is to present an exhaustive investigation for the forced vibration of a buckled beam with quadratic and cubic nonlinearities in the equations of motion. Buckled beams play an important role in the design of machines and structures. Buckled beams have received a great deal of attention from various scholars also due to their complex nonlinear behaviors [1]. For example, the nonlinear modal interaction [2] and the internal resonance [3] are arising out of commensurable relationships of frequencies for specific values of the system parameters. Furthermore, in the presence of external excitation, the internal resonance can have possible influence on the buckled beam behavior, which needs to be studied [4].

Lacarbonara et al. [5] studied the frequency-response curves of a primary resonance of a buckled beam. The authors found that the response curves obtained using a singlemode approximation are in disagreement with those obtained by the experiment. Emam and Nayfeh [6] focused on the dynamics of a buckled beam subject to a primary-resonance excitation via the Galerkin truncation. They revealed that using a single-mode truncation leads to errors in the static and dynamic problems. Nayfeh and Balachandran pointed out that those systems are characterized by quadratic nonlinearities which may lead to two-to-one and combination autoparametric resonances [7]. Chin and Nayfeh considered a hinged-clamped beam with a cubic nonlinearity and the three-to-one ratio of the second and first natural frequencies [8]. The authors found that the beam exhibits a threeto-one internal resonance resulting in an energy exchange between these modes. Based on an initially buckled beam with clamped ends, Afaneh and Ibrahim found that the first mode transfers energy to the second mode in the presence of the 1:1 internal resonance [9]. Chin et al. analyzed the response of a buckled beam possessing a two-to-one internal resonance to a principal parametric resonance of the higher mode [10]. The authors found that the response curves exhibit the double-jump and the saturation phenomenon. Based on a simply-supported beam under a vertical concentrated load, 
$Q(X, T)=F(X) \cos (\Omega T)$

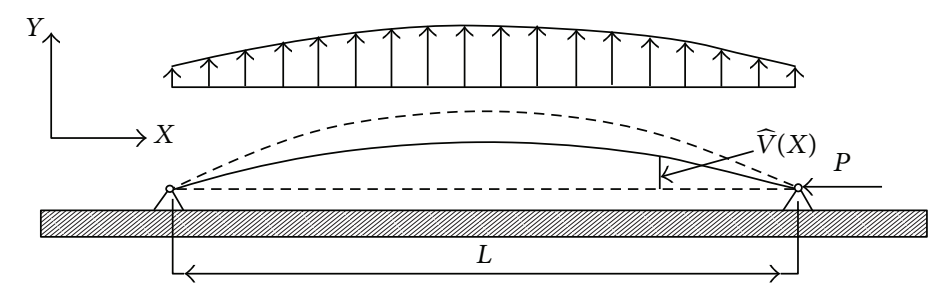

FIGURE 1: A buckled beam with external harmonic excitation.

Machado and Saravia [11] analyzed the resonance responses in internal resonance conditions of the kind $2: 3: 1$. Emam and Nayfeh [12] focused on a primary-resonance excitation in the presence of 1-1 and 3-1 internal resonances for a buckled beam with fixed ends. The investigation shows that the interacting modes are nonlinearly coupled. It is worth noting that all above-mentioned researchers assumed that the buckled beams under their consideration are elastic, and did not account for any internal damping factors. On the other hand, it is well known that the dissipation of energy using viscoelastic damping materials within vibrating structures can reduce noise and vibration. Due to their importance in the design of the continuous structures and systems, the effects of internal damping have been widely studied for the last two decades. A comprehensive overview can be found in $[13,14]$. Very recently, Galuppi and Royer-Carfagni found that there are noteworthy differences between the quasielastic and the full viscoelastic model [15]. So far, from the literature survey, it is visible that the buckling and vibration behavior of the viscoelastic beam with internal resonances has not been investigated. To address the lack of research in this aspect, this work is devoted to studying forced vibration of a viscoelastic buckled beams in the presence of the 2:1 internal resonance.

A nonlinear phenomenon has been found in the dynamic analysis for the system with quadratic nonlinearities resulting in a two-to-one internal resonance. More precisely, it will be shown that there are regions in the frequencyresponse curves of first primary resonance without any stable solution. This phenomenon was first found by Nayfeh and his coworkers by a two-degree-of-freedom discrete system with quadratic nonlinearities [16, 17]. Alasty and Shabani found that the nonperiodic regions exist in discrete springpendulums [18]. The authors found that the solutions are quasi-periodic in most points of the unstable periodic regions and only in special cases the chaotic solutions may occur. Very recently, based on the dynamics of a pipe conveying fluid in the supercritical flow speed regime with external and internal resonance, Chen and his coworkers [19] found that the non-periodic region phenomena occur in a gyroscopic system. From the review of literature, it is found that the study of the nonperiodic region in the internal resonance in the area of continua systems, such as buckled beams, has not yet been explored so far. Hence, the present investigation proposes to investigate the same.

In this work, an analytical treatment is performed to investigate nonlinear dynamics of the viscoelastic buckled beam with external and two-to-one internal resonance around the stable curved equilibrium configuration. The stable steady-state periodic response curves from the multiple scales method are qualitatively and quantitatively confirmed by Runge-Kutta method. The unstable regions are discussed by numerical simulations. The present paper is organized into six sections. Section 2 describes the modeling of a buckled beam subjected to an external distributed harmonic excitation. Steady-state solutions and their stability are discussed in Section 3. Section 4 presents the results of approximate solutions for illustrative examples. The numerical verifications are presented in Section 5. Section 6 ends the paper with concluding remarks.

\section{Mathematical Model}

Consider a slender beam hinged to a base undergoing harmonic motion $F(X) \cos \Omega T$, as illustrated schematically in Figure 1. The beam is modeled as an Euler-Bernoulli beam which is made of the viscoelastic material. The equation of transverse motion of the beam with external harmonic excitation is given by $[10,19]$

$$
\begin{aligned}
m V,_{T T} & +P V,_{X X}+E I V,_{X X X X}+I \Lambda V,_{X X X X T} \\
= & \frac{E A}{2 L} V,_{X X} \int_{0}^{L} V,_{X}^{2} \mathrm{~d} X+\frac{A \Lambda}{L} V,_{X X} \\
& \times \int_{0}^{L} V,_{X} V,_{X T} \mathrm{~d} X+F(X) \cos (\Omega T),
\end{aligned}
$$

where $X$ is the neutral axis coordinate along the undeflected beam and $T$ is the time. A comma preceding $X$ or $T$ denotes partial derivatives with respect to $X$ or $T . V(X, T)$ is the transverse displacement. $L$ is the length, $A$ is the cross-section area, $m$ is the mass per unit length, $I$ is the area moment of inertial, and $P$ is the axial load. The viscoelastic material is constituted by the Kelvin relation with Young's modulus $E$ and the viscoelastic damping coefficient $\Lambda$ [19]. The effect of the external damping is neglected here. The beam is simply supported at both ends

$$
\begin{gathered}
V(0, T)=V(L, T)=0, \\
V,_{X X}(0, T)=V,_{X X}(L, T)=0 .
\end{gathered}
$$


Incorporating the following dimensionless quantities:

$$
\begin{gathered}
v=\frac{V}{L}, \quad x=\frac{X}{L}, \quad t=\frac{T}{L} \sqrt{\frac{P}{m}}, \\
f(x)=\frac{L}{P} F(x), \quad \omega=\Omega L \sqrt{\frac{m}{P}}, \\
\alpha=\frac{I \Lambda}{L^{3} \sqrt{m P}}, \quad k_{1}^{2}=\frac{E A}{P}, \quad k_{f}^{2}=\frac{E I}{P L^{2}},
\end{gathered}
$$

the equation of motion and the boundary conditions can be nondimensionalized as

$$
\begin{gathered}
v_{t t}+v_{x_{x x}}+k_{f}^{2} v,_{x x x x}+\alpha v_{x x x x t} \\
=f(x) \cos (\omega t)+\frac{k_{1}^{2}}{2} v,_{x x} \\
\times \int_{0}^{1} v_{x}^{2} \mathrm{~d} x+\frac{\alpha k_{1}^{2}}{k_{f}^{2}} v,_{x x} \int_{0}^{1} v_{x} v_{x t} \mathrm{~d} x, \\
v(0, t)=v(1, t)=0, \\
v_{r_{x x}}(0, t)=v,_{x x}(1, t)=0 .
\end{gathered}
$$

If the axial load is larger than the critical value, the straight equilibrium configuration becomes unstable. The static buckling deflection $\widehat{v}(x)$ is governed by

$$
\widehat{v}^{\prime \prime}+k_{f}^{2} \widehat{v}^{\prime \prime \prime \prime}=\frac{k_{1}^{2}}{2} \widehat{v}^{\prime \prime} \int_{0}^{1}\left(\widehat{v}^{\prime}\right)^{2} \mathrm{~d} x .
$$

The first buckling mode of hinged-hinged beams is the only stable equilibrium position [12]. One assumes a solution in the form

$$
\widehat{v}(x)=A_{S} \sin (\pi x) .
$$

Substituting (7) into (6), one obtains the equilibrium solution

$$
A_{S}=\frac{2}{k_{1} \pi} \sqrt{1-k_{f}^{2} \pi^{2}} .
$$

Therefore, the transformation $v(x, t) \leftrightarrow \widehat{v}(x)+v(x, t)$ in (4) yields the governing equation of motion measured from the first buckling mode

$$
\begin{aligned}
v_{t t} & +\pi^{2} k_{f}^{2} v_{x x}+k_{f}^{2} v_{x x x x}+\alpha v,_{x x x x t} \\
& =-\frac{\alpha k_{1}^{2}}{k_{f}^{2}}\left[v_{x x}-A_{S} \pi^{2} \sin (\pi x)\right]
\end{aligned}
$$

$$
\begin{aligned}
& \times \int_{0}^{1}\left[v+A_{S} \sin (\pi x)\right] v_{x x t} \mathrm{~d} x \\
& +\frac{k_{1}^{2}}{2}\left[v_{x x}-A_{S} \pi^{2} \sin (\pi x)\right] \\
& \times \int_{0}^{1}\left[v_{x_{x}}^{2}+2 \pi^{2} A_{S} v \sin (\pi x)\right] \mathrm{d} x \\
& +f(x) \cos (\omega t) .
\end{aligned}
$$

\section{Schemes of Solution}

3.1. Galerkin Discretization. The Galerkin truncation will be proposed to discretize the equation of motion of a simply supported viscoelastic buckled beam into ordinary differential equations. Suppose that the transverse displacement $v(x, t)$ is approximated by

$$
v(x, t)=\sum_{j=1}^{n} \phi_{j}(x) q_{j}(t),
$$

where $\phi_{j}(x)(j=1,2, \ldots, n)$ is the $j$ th eigenfunction of the free undamped vibration of the beam with the hinged-hinged boundary conditions; namely, $\phi_{j}(x)=\sin (j \pi x)$, and $q_{j}(t)$ $(j=1,2, \ldots, n)$ is the $j$ th modal coordinates.

The second-order Galerkin truncation is investigated first, as the effect of higher-order mode will be discussed in Section 3.5. Substituting (10) (with $n=2$ ) into (9), multiplying the resulting equation by weighted function $\phi_{j}(x)(j=1,2)$, and integrating the product from 0 to 1 yield

$$
\begin{aligned}
\ddot{q}_{1}+ & \alpha \pi^{4} \dot{q}_{1}+\frac{1}{2} k_{1}^{2} \pi^{4} A_{S}^{2} q_{1} \\
= & 2 \cos (\omega t) \int_{0}^{1} f(x) \sin (\pi x) \mathrm{d} x \\
& -\frac{1}{2} k_{1}^{2} \pi^{4}\left\{A_{S} q_{1}^{2}+\frac{1}{2}\left(A_{S}+q_{1}\right)\right. \\
& \left.\times\left[q_{1}^{2}+4 q_{2}^{2}+\frac{2 \alpha}{k_{f}^{2}}\left(A_{S} \dot{q}_{1}+q_{1} \dot{q}_{1}+4 q_{2} \dot{q}_{2}\right)\right]\right\} \\
\ddot{q}_{2}+16 \alpha \pi^{4} \dot{q}_{2}+12 \pi^{4} k_{f}^{2} q_{2} & \\
= & 2 \cos (\omega t) \int_{0}^{1} f(x) \sin (2 \pi x) \mathrm{d} x \\
& -k_{1}^{2} \pi^{4} q_{2}\left[2 A_{S} q_{1}+q_{1}^{2}+4 q_{2}^{2}\right. \\
& \left.+\frac{2 \alpha}{k_{f}^{2}}\left(A_{S} \dot{q}_{1}+q_{1} \dot{q}_{1}+4 q_{2} \dot{q}_{2}\right)\right] .
\end{aligned}
$$

3.2. 2:1 Internal Resonance. The method of multiple scales will be employed to seek an approximate solution to (11). 
Introduce the fast and slow time scales $T_{0}=t$ and $T_{1}=\varepsilon t$. The approximate expansions of the solutions to (11) are assumed to be

$$
\begin{aligned}
& q_{1}\left(T_{0}, T_{1}\right)=\varepsilon q_{11}\left(T_{0}, T_{1}\right)+\varepsilon^{2} q_{12}\left(T_{0}, T_{1}\right)+\cdots, \\
& q_{2}\left(T_{0}, T_{1}\right)=\varepsilon q_{21}\left(T_{0}, T_{1}\right)+\varepsilon^{2} q_{22}\left(T_{0}, T_{1}\right)+\cdots,
\end{aligned}
$$

where $\varepsilon$ is a small nondimensional bookkeeping parameter that is used to distinguish different orders of magnitude. At the end of analysis, the bookkeeping parameter's value is set to be equal to unity. For weak external excitations, $\alpha$ and $f$ are scaled as $\alpha \leftrightarrow \varepsilon \alpha$ and $f \leftrightarrow \varepsilon^{2} f$. Substitution of (12) into (11) and equalization of coefficients of $\varepsilon^{0}$ and $\varepsilon^{1}$ in the resulting equations lead to.

Order $\varepsilon^{0}$

$$
\begin{gathered}
q_{11, T_{0} T_{0}}+\frac{1}{2} k_{1}^{2} \pi^{4} A_{S}^{2} q_{11}=0, \\
q_{21, T_{0} T_{0}}+12 \pi^{4} k_{f}^{2} q_{21}=0 .
\end{gathered}
$$

Order $\varepsilon^{1}$

$$
\begin{aligned}
q_{12} T_{0} T_{0} & +\frac{1}{2} k_{1}^{2} \pi^{4} A_{S}^{2} q_{12} \\
= & 2 \cos (\omega t) \int_{0}^{1} f(x) \sin (\pi x) \mathrm{d} x \\
& -k_{1}^{2} \pi^{4} A_{S}\left(\frac{3}{4} q_{11}^{2}+q_{21}^{2}\right) \\
& -\alpha \pi^{4}\left(1+\frac{k_{1}^{2} A_{S}^{2}}{2 k_{f}^{2}}\right) q_{11} T_{0}-2 q_{11}{ }^{\prime} T_{0} T_{1}, \\
q_{22}, T_{0} T_{0} & +12 \pi^{4} k_{f}^{2} q_{22} \\
= & 2 \cos (\omega t) \int_{0}^{1} f(x) \sin (2 \pi x) \mathrm{d} x \\
& -2 k_{1}^{2} \pi^{4} A_{S} q_{11} q_{21}-16 \alpha \pi^{4} q_{21}, T_{0}-2 q_{21},_{0} T_{1} .
\end{aligned}
$$

Equation (13) defines a two-degree-of-freedom linear system. Its two natural frequencies are

$$
\omega_{1}=\min \left(\omega_{a}, \omega_{b}\right), \quad \omega_{2}=\max \left(\omega_{a}, \omega_{b}\right),
$$

where $\omega_{a}=k_{1} \pi^{2} A_{S} / \sqrt{2}$ and $\omega_{b}=2 \sqrt{3} \pi^{2} k_{f}$. Figure 2 shows variation of the nondimensional natural frequencies with the nondimensional parameter $k_{f}$. As can be noted from the figure, 2:1 internal resonance will be activated when $k_{f}$ is near 0.06366 or 0.20132 . form:

The solution of (13) can be expressed in the following

$$
\begin{aligned}
& q_{11}\left(T_{0}, T_{1}\right)=Y_{1}\left(T_{1}\right) e^{i \omega_{a} T_{0}}+\mathrm{cc}, \\
& q_{21}\left(T_{0}, T_{1}\right)=Y_{2}\left(T_{1}\right) e^{i \omega_{b} T_{0}}+\mathrm{cc},
\end{aligned}
$$

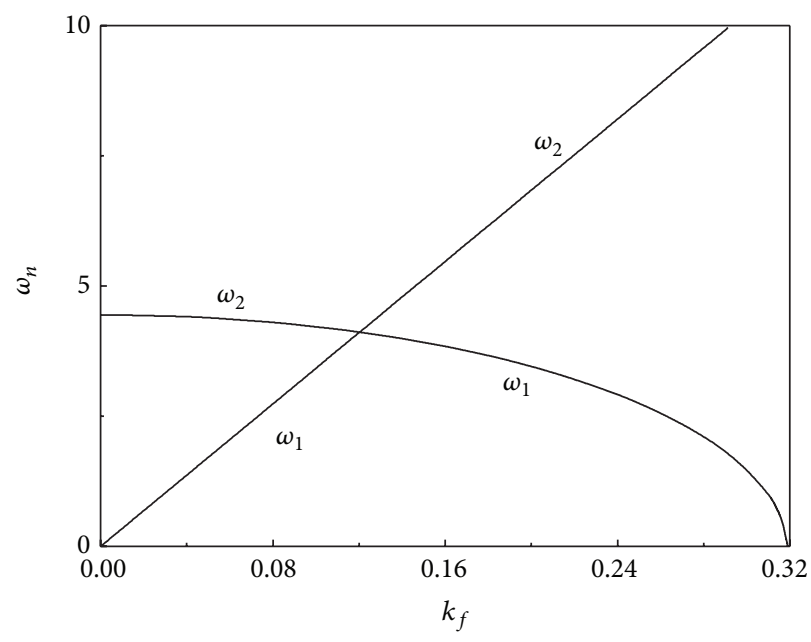

FIGURE 2: Variation of the natural frequencies of a buckled beam with the parameter $k_{f}$.

where $\mathrm{cc}$ stands for the complex conjugate of the preceding terms. Substitution of (16) into (14) yields

$$
\begin{aligned}
q_{12},_{0} T_{0} & +\omega_{a}^{2} q_{12} \\
= & e^{i \omega T_{0}} \int_{0}^{1} f(x) \sin (\pi x) \mathrm{d} x-k_{1}^{2} \pi^{4} A_{S} Y_{2}^{2} e^{2 i \omega_{b} T_{0}} \\
& -\alpha \pi^{4}\left(1+\frac{k_{1}^{2} A_{S}^{2}}{2 k_{f}^{2}}\right) i \omega_{a} Y_{1} e^{i \omega_{a} T_{0}} \\
& -2 i \omega_{a} Y_{1}^{\prime} e^{i \omega_{a} T_{0}}+\mathrm{cc}+\mathrm{NST} \\
q_{22} T_{0} T_{0} & +\omega_{b}^{2} q_{22} \\
= & e^{i \omega T_{0}} \int_{0}^{1} f(x) \sin (2 \pi x) \mathrm{d} x \\
& -2 k_{1}^{2} \pi^{4} A_{S} Y_{1} \bar{Y}_{2} e^{i\left(\omega_{a}-\omega_{b}\right) T_{0}} \\
& -16 \alpha \pi^{4} i \omega_{b} Y_{2} e^{i \omega_{b} T_{0}} \\
& -2 i \omega_{b} Y_{2}^{\prime} e^{i \omega_{b} T_{0}}+\mathrm{cc}+\mathrm{NST}
\end{aligned}
$$

where NST stands for all the other nonsecular terms. If $\omega_{a}=$ $2 \omega_{b}$, some complex nonlinear behaviors may be observed due to the resulting nonlinear secular terms. Under this condition, one obtains $\omega_{1}=\omega_{b}, \omega_{2}=\omega_{a}$ and $k_{f}=0.06366$. The chief aim of the present work is to investigate primary resonance in the presence of $2: 1$ internal resonance.

3.3. First Primary Resonance. When $f(x)=b \sin (2 \pi x)$, the primary resonance of the first mode in the presence of 2:1 internal resonance will be investigated. Introduce the detuning parameters $\sigma$ and $\sigma_{0}$ to describe the nearness of $\omega$ to $\omega_{1}$ and $\omega_{2}$ to $2 \omega_{1}$, respectively. Thus,

$$
\omega_{2}=2 \omega_{1}+\varepsilon \sigma_{0}, \quad \omega=\omega_{1}+\varepsilon \sigma .
$$


Substitution of (18) into (17) and equalization of coefficients of $e^{i \omega_{1} T_{0}}$ and $e^{i \omega_{2} T_{0}}$ on both sides of the resulting equation lead to

$$
\begin{gathered}
-k_{1}^{2} \pi^{4} A_{S} Y_{2}^{2} e^{-i \sigma_{0} T_{1}}-\alpha \pi^{4}\left(1+\frac{k_{1}^{2} A_{S}^{2}}{2 k_{f}^{2}}\right) i \omega_{2} Y_{1}-2 i \omega_{2} Y_{1}^{\prime}=0, \\
\frac{b}{2} e^{i \sigma T_{1}}-2 k_{1}^{2} \pi^{4} A_{S} Y_{1} \bar{Y}_{2} e^{i \sigma_{0} T_{1}}-16 \alpha \pi^{4} i \omega_{1} Y_{2}-2 i \omega_{1} Y_{2}^{\prime}=0 .
\end{gathered}
$$

Express the solution to (19) in the polar form

$$
\begin{aligned}
& Y_{1}\left(T_{1}\right)=\frac{1}{2} a_{1}\left(T_{1}\right) e^{i \gamma_{1}\left(T_{1}\right)}, \\
& Y_{2}\left(T_{1}\right)=\frac{1}{2} a_{2}\left(T_{1}\right) e^{i \gamma_{2}\left(T_{1}\right)},
\end{aligned}
$$

where $a_{n}$ and $\gamma_{n}$ are the real valued amplitude and phase respectively. Substituting (20) into (19) and separating the resulting equation into real and imaginary parts yield

$$
\begin{aligned}
a_{1}^{\prime}= & \frac{1}{4} \omega_{2}^{-1} a_{2}{ }^{2} k_{1}^{2} \pi^{4} A_{S} \sin \left(2 \theta_{2}-\theta_{1}\right) \\
& -\frac{1}{2} a_{1} \alpha \pi^{4}\left(1+\frac{k_{1}^{2} A_{S}^{2}}{2 k_{f}^{2}}\right), \\
a_{2}^{\prime}= & \frac{b}{2} \omega_{1}^{-1} \sin \theta_{2}-\frac{1}{2} \omega_{1}^{-1} a_{1} a_{2} k_{1}^{2} \pi^{4} A_{S} \sin \left(2 \theta_{2}-\theta_{1}\right) \\
& -8 \alpha \pi^{4} a_{2}, \\
a_{1} \theta_{1}^{\prime}= & a_{1}\left(2 \sigma-\sigma_{0}\right)-\frac{1}{4} \omega_{2}^{-1} a_{2}^{2} k_{1}^{2} \pi^{4} A_{S} \cos \left(2 \theta_{2}-\theta_{1}\right), \\
a_{2} \theta_{2}^{\prime}= & \frac{b}{2} \omega_{1}^{-1} \cos \theta_{2}-\frac{1}{2} \omega_{1}^{-1} a_{1} a_{2} k_{1}^{2} \pi^{4} A_{S} \cos \left(2 \theta_{2}-\theta_{1}\right) \\
& +a_{2} \sigma,
\end{aligned}
$$

where $\theta_{1}=2 \sigma T_{1}-\sigma_{0} T_{1}-\gamma_{1}$ and $\theta_{2}=\sigma T_{1}-\gamma_{2}$. Steady-state responses occur when $a_{n}$ and $\theta_{n}$ are constants. Eliminating $\theta_{1}, \theta_{2}, a_{1}$ from (21), the frequency-response relationship is obtained

$$
\begin{aligned}
\frac{b^{2}}{4 \omega_{1}^{2}}= & \left\{\frac{\omega_{2} \alpha k_{1}^{2} \pi^{8}\left(1+k_{1}^{2} A_{S}^{2} / 2 k_{f}^{2}\right) a_{2}^{3}}{2 \omega_{1}\left[\alpha^{2} \pi^{8}\left(1+k_{1}^{2} A_{S}^{2} / 2 k_{f}^{2}\right)^{2}+4\left(2 \sigma-\sigma_{0}\right)^{2}\right]}\right. \\
& \left.+8 \alpha \pi^{4} a_{2}\right\}^{2} \\
& +\left\{\frac{\omega_{1}\left[\alpha^{2} \pi^{8}\left(1+k_{1}^{2} A_{S}^{2} / 2 k_{f}^{2}\right)^{2}+4\left(2 \sigma-\sigma_{0}\right)^{2}\right]}{\omega_{2}^{2} \pi^{4}\left(2 \sigma-\sigma_{0}\right) a_{2}^{3}}\right. \\
& \left.-\sigma a_{2}\right\}^{2} .
\end{aligned}
$$

Then the frequency-response relationship in the $a_{1}$ mode can be obtained

$$
a_{1}=\frac{k_{1} \pi^{2} a_{2}^{2}}{\sqrt{2 \alpha^{2} \pi^{8}\left(1+k_{1}^{2} A_{S}^{2} / 2 k_{f}^{2}\right)^{2}+8\left(2 \sigma-\sigma_{0}\right)^{2}}} .
$$

The stability of the steady-state responses can be determined by the Routh-Hurwitz criterion. The real parts of eigenvalues of the Jacobian matrix of (21) reveal the stability of the fixed point.

3.4. Second Primary Resonance. When $f(x)=b \sin (\pi x)$, the primary resonance of the second mode in the presence of $2: 1$ internal resonance will be investigated. In this case, the frequency relations for the internal resonance and second primary resonance are introduced

$$
\omega_{2}=2 \omega_{1}+\varepsilon \sigma_{0}, \quad \omega=\omega_{2}+\varepsilon \sigma,
$$

where $\sigma$ and $\sigma_{0}$ are the detuning parameters. Substituting (24) into (17) and equating the coefficients of $e^{i \omega_{1} T_{0}}$ and $e^{i \omega_{2} T_{0}}$ on both sides, one obtains

$$
\begin{aligned}
\frac{b}{2} e^{i \sigma T_{1}} & -k_{1}^{2} \pi^{4} A_{S} Y_{2}^{2} e^{-i \sigma_{0} T_{1}}-\alpha \pi^{4}\left(1+\frac{k_{1}^{2} A_{S}^{2}}{2 k_{f}^{2}}\right) i \omega_{2} Y_{1} \\
& -2 i \omega_{2} Y_{1}^{\prime}=0, \\
& -2 k_{1}^{2} \pi^{4} A_{S} Y_{1} \bar{Y}_{2} e^{i \sigma_{0} T_{1}}-16 \alpha \pi^{4} i \omega_{1} Y_{2}-2 i \omega_{1} Y_{2}^{\prime}=0 .
\end{aligned}
$$

The polar transformations for $Y_{1}$ and $Y_{2}$ are introduced in (20). Substituting (20) into (25) and separating the resulting equation into real and imaginary parts yield

$$
\begin{aligned}
a_{1}^{\prime}= & \frac{b}{2} \omega_{2}^{-1} \sin \theta_{1}+\frac{1}{4} \omega_{2}^{-1} a_{2}^{2} k_{1}^{2} \pi^{4} A_{S} \sin \left(2 \theta_{2}-\theta_{1}\right) \\
& -\frac{1}{2} a_{1} \alpha \pi^{4}\left(1+\frac{k_{1}^{2} A_{S}^{2}}{2 k_{f}^{2}}\right) \\
a_{2}^{\prime}= & -\frac{1}{2} \omega_{1}^{-1} a_{1} a_{2} k_{1}^{2} \pi^{4} A_{S} \sin \left(2 \theta_{2}-\theta_{1}\right)-8 \alpha \pi^{4} a_{2}, \\
& a_{1} \theta_{1}^{\prime}=\frac{b}{2} \omega_{2}^{-1} \cos \theta_{1}+a_{1} \sigma \\
a_{2} \theta_{2}^{\prime}=- & \frac{1}{2} \omega_{1}^{-1} a_{1} a_{2} k_{1}^{2} \pi^{4} A_{S} \cos \left(2 \theta_{2}-\theta_{1}\right)+\frac{1}{2} a_{2}\left(\sigma+\sigma_{0}\right)
\end{aligned}
$$

where $\theta_{1}=\sigma T_{1}-\gamma_{1}$ and $\theta_{2}=0.5 \sigma T_{1}+0.5 \sigma_{0} T_{1}-\gamma_{2}$. The steady-state solutions are obtained by setting the right-hand side of (26) equal to zero. There are two possible solutions. The first is a single-mode $\left(a_{2}=0\right)$ steady-state solution given 
by (27). This is the solution of local linearization (as indicated by the subscript "l")

$$
\begin{gathered}
a_{1}=a_{1 l}=\frac{b}{\omega_{2} \sqrt{\alpha^{2} \pi^{8}\left(1+k_{1}^{2} A_{S}^{2} / 2 k_{f}^{2}\right)^{2}+4 \sigma^{2}}}, \\
a_{2}=a_{2 l}=0 .
\end{gathered}
$$

The other possibility is coupled mode $\left(a_{2} \neq 0\right)$ steady-state solution. This is the nonlinear solution (as indicated by the subscript “ $n$ ")

$$
\begin{gathered}
a_{1}=a_{1 n}=\frac{k_{f}}{\pi k_{1}} \sqrt{\frac{3\left(\sigma+\sigma_{0}\right)^{2}+768 \alpha^{2} \pi^{8}}{1-\pi^{2} k_{f}^{2}}}, \\
a_{2}=a_{2 n}=\frac{1}{\pi^{2}} \sqrt{\frac{\sqrt{6} \Gamma_{1} \pm\left(4 k_{1}^{2} k_{f}^{2} b^{2}-6 \pi^{8} \Gamma_{2}^{2}\right)^{1 / 2}}{A_{S} k_{f} k_{1}^{3}}},
\end{gathered}
$$

where

$$
\begin{gathered}
\Gamma_{1}=4 k_{f}^{2} \sigma\left(\sigma+\sigma_{0}\right)-16 \pi^{8} \alpha^{2}\left(2 k_{f}^{2}+A_{S}^{2} k_{1}^{2}\right), \\
\Gamma_{2}=\alpha\left[\left(66 \sigma+2 \sigma_{0}\right) k_{f}^{2}+A_{S}^{2} k_{1}^{2}\left(\sigma+\sigma_{0}\right)\right] .
\end{gathered}
$$

The stability of the nontrivial state can be determined by the Routh-Hurwitz criterion, but it is not suitable for the single-mode state. To determine the stability of the local linear solution, an alternative Cartesian formulation for the complex amplitude equations will be used as follows:

$$
\begin{aligned}
& Y_{1}\left(T_{1}\right)=\frac{1}{2}\left[x_{1}\left(T_{1}\right)-i y_{1}\left(T_{1}\right)\right] e^{i v_{1} T_{1}}, \\
& Y_{2}\left(T_{1}\right)=\frac{1}{2}\left[x_{2}\left(T_{1}\right)-i y_{2}\left(T_{1}\right)\right] e^{i v_{2} T_{1}},
\end{aligned}
$$

where $v_{1}=\sigma$ and $v_{2}=(1 / 2)\left(\sigma+\sigma_{0}\right)$. Substituting the new definition (30) into (25), one finally has

$$
\begin{aligned}
x_{1}^{\prime}= & \frac{k_{1}^{2} \pi^{4} A_{S}}{2 \omega_{2}} x_{2} y_{2}-\frac{1}{2} \alpha \pi^{4}\left(1+\frac{k_{1}^{2} A_{S}^{2}}{2 k_{f}^{2}}\right) x_{1}-v_{1} y_{1} \\
y_{1}^{\prime}= & \frac{b}{2 \omega_{2}}-\frac{k_{1}^{2} \pi^{4} A_{S}}{4 \omega_{2}}\left(x_{2}^{2}-y_{2}^{2}\right)-\frac{1}{2} \alpha \pi^{4}\left(1+\frac{k_{1}^{2} A_{S}^{2}}{2 k_{f}^{2}}\right) y_{1} \\
& +v_{1} x_{1}, \\
x_{2}^{\prime}= & \frac{k_{1}^{2} \pi^{4} A_{S}}{2 \omega_{1}}\left(x_{2} y_{1}-x_{1} y_{2}\right)-8 \alpha \pi^{4} x_{2}-v_{2} y_{2} \\
y_{2}^{\prime}= & v_{2} x_{2}-\frac{k_{1}^{2} \pi^{4} A_{S}}{2 \omega_{1}}\left(x_{1} x_{2}+y_{1} y_{2}\right)-8 \alpha \pi^{4} y_{2} .
\end{aligned}
$$

Then the stability of the local linear solution is determined in a similar way to the previous case.
3.5. Influence of Higher Truncation Order. In the presence of $2: 1$ internal resonance, the effect of higher truncation order on the dynamic responses of the buckled beam will be discussed.

Substituting (10) (with $n=4$ ) into (9), multiplying the resulting equation by weighted function $\phi_{j}(x)(j=1,2,3,4)$, and integrating the product from 0 to 1 yield

$$
\begin{aligned}
& \ddot{q}_{1}+ \alpha \pi^{4} \dot{q}_{1}+\frac{1}{2} k_{1}^{2} \pi^{4} A_{S}^{2} q_{1} \\
&= 2 \cos (\omega t) \int_{0}^{1} f(x) \sin (\pi x) \mathrm{d} x-\frac{1}{2} k_{1}^{2} \pi^{4} A_{S} q_{1}^{2} \\
&-\frac{1}{4} k_{1}^{2} \pi^{4}\left(A_{S}+q_{1}\right) \\
& \quad\left[q_{1}^{2}+4 q_{2}^{2}+9 q_{3}^{2}+16 q_{4}^{2}+\frac{2 \alpha}{k_{f}^{2}}\right. \\
&\left.\times\left(A_{S} \dot{q}_{1}+q_{1} \dot{q}_{1}+4 q_{2} \dot{q}_{2}+9 q_{3} \dot{q}_{3}+16 q_{4} \dot{q}_{4}\right)\right] \\
& \ddot{q}_{2}+16 \alpha \pi^{4} \dot{q}_{2}+12 \pi^{4} k_{f}^{2} q_{2} \\
&=2 \cos (\omega t) \int_{0}^{1} f(x) \sin (2 \pi x) \mathrm{d} x \\
& \quad k_{1}^{2} \pi^{4} q_{2}\left[2 A_{S} q_{1}+q_{1}^{2}+4 q_{2}^{2}+9 q_{3}^{2}+16 q_{4}^{2}+\frac{2 \alpha}{k_{f}^{2}}\right. \\
& \times\left(A_{S} \dot{q}_{1}+q_{1} \dot{q}_{1}+4 q_{2} \dot{q}_{2}\right. \\
&\left.\left.+9 q_{3} \dot{q}_{3}+16 q_{4} \dot{q}_{4}\right)\right],
\end{aligned}
$$$$
\ddot{q}_{3}+81 \alpha \pi^{4} \dot{q}_{3}+72 \pi^{4} k_{f}^{2} q_{3}
$$$$
=2 \cos (\omega t) \int_{0}^{1} f(x) \sin (3 \pi x) \mathrm{d} x
$$$$
-\frac{9}{4} k_{1}^{2} \pi^{4} q_{3}\left[2 A_{S} q_{1}+q_{1}^{2}+4 q_{2}^{2}+9 q_{3}^{2}+16 q_{4}^{2}+\frac{2 \alpha}{k_{f}^{2}}\right.
$$$$
\times\left(A_{S} \dot{q}_{1}+q_{1} \dot{q}_{1}+4 q_{2} \dot{q}_{2}\right.
$$$$
\left.\left.+9 q_{3} \dot{q}_{3}+16 q_{4} \dot{q}_{4}\right)\right]
$$

$$
\begin{gathered}
\ddot{q}_{4}+256 \alpha \pi^{4} \dot{q}_{4}+240 \pi^{4} k_{f}^{2} q_{4} \\
=2 \cos (\omega t) \int_{0}^{1} f(x) \sin (4 \pi x) \mathrm{d} x \\
-4 k_{1}^{2} \pi^{4} q_{4}\left[2 A_{S} q_{1}+q_{1}^{2}+4 q_{2}^{2}+9 q_{3}^{2}+16 q_{4}^{2}+\frac{2 \alpha}{k_{f}^{2}}\right. \\
\times\left(A_{S} \dot{q}_{1}+q_{1} \dot{q}_{1}+4 q_{2} \dot{q}_{2}\right. \\
\left.\left.+9 q_{3} \dot{q}_{3}+16 q_{4} \dot{q}_{4}\right)\right] .
\end{gathered}
$$


There exist four different natural frequencies

$$
\begin{gathered}
\omega_{a}=\pi \sqrt{2\left(1-k_{f}^{2} \pi^{2}\right)}, \quad \omega_{b}=\sqrt{12} \pi^{2} k_{f}, \\
\omega_{c}=\sqrt{72} \pi^{2} k_{f}, \quad \omega_{d}=\sqrt{240} \pi^{2} k_{f} .
\end{gathered}
$$

Under the condition of $\omega_{a}=2 \omega_{b}$, namely, $2: 1$ internal resonance, one obtains

$$
\begin{gathered}
\omega_{1}=\sqrt{12} \pi^{2} k_{f}, \quad \omega_{2}=\pi \sqrt{2\left(1-k_{f}^{2} \pi^{2}\right)}, \\
\omega_{3}=\sqrt{72} \pi^{2} k_{f}, \quad \omega_{4}=\sqrt{240} \pi^{2} k_{f} .
\end{gathered}
$$

As compared with the second-order Galerkin truncation, the third and fourth natural frequencies are added and the first two natural frequencies have no change.

The first and second primary resonances under the harmonic load $f(x)=b \sin (2 \pi x)$ and $f(x)=b \sin (\pi x)$ are investigated, respectively. Based on the orthogonal property of the trigonometric functions, substitution of $f(x)$ into (10) leads to

$$
\begin{gathered}
\ddot{q}_{3}+81 \alpha \pi^{4} \dot{q}_{3}+72 \pi^{4} k_{f}^{2} q_{3} \\
=-\frac{9}{4} k_{1}^{2} \pi^{4} q_{3}\left[2 A_{S} q_{1}+q_{1}^{2}+4 q_{2}^{2}+9 q_{3}^{2}+16 q_{4}^{2}+\frac{2 \alpha}{k_{f}^{2}}\right. \\
\times\left(A_{S} \dot{q}_{1}+q_{1} \dot{q}_{1}+4 q_{2} \dot{q}_{2}\right. \\
\left.\left.+9 q_{3} \dot{q}_{3}+16 q_{4} \dot{q}_{4}\right)\right], \\
\ddot{q}_{4}+256 \alpha \pi^{4} \dot{q}_{4}+240 \pi^{4} k_{f}^{2} q_{4} \\
=-4 k_{1}^{2} \pi^{4} q_{4}\left[\begin{array}{c}
2 A_{S} q_{1}+q_{1}^{2}+4 q_{2}^{2}+9 q_{3}^{2}+16 q_{4}^{2}+\frac{2 \alpha}{k_{f}^{2}} \\
\times\left(A_{S} \dot{q}_{1}+q_{1} \dot{q}_{1}+4 q_{2} \dot{q}_{2}\right. \\
\left.\left.+9 q_{3} \dot{q}_{3}+16 q_{4} \dot{q}_{4}\right)\right] .
\end{array}\right.
\end{gathered}
$$

There is no stimulus for modal coordinates $q_{3}$ and $q_{4}$. In addition, it could be deduced that the $q_{3}$ and $q_{4}$ will not be activated by internal resonance between the first two modes, since the added natural frequencies and the first two natural frequencies are incommensurable. The steady-state responses for $q_{3}$ and $q_{4}$ only have trivial solutions. Similar results will be obtained for higher truncation order.

\section{Case Studies}

In this section, approximate analytical results will be illustrated by numerical examples. LC4 superduralumin is mainly applied to the structure suffered from large load in aircraft. In the following numerical case, a superduralumin beam with circular cross section will be considered. Its physical parameters are

$$
\begin{gathered}
L=1 \mathrm{~m}, \quad A=3.14159 \mathrm{e}^{-4} \mathrm{~m}^{2}, \\
E=74 \mathrm{Gpa}, \quad I=7.85398 \mathrm{e}^{-9} \mathrm{~m}^{4} .
\end{gathered}
$$

Perfect 2:1 internal resonance is activated when $k_{f}=$ 0.063662 . In this case,

$$
P=\frac{E I}{k_{f}^{2} L^{2}}=143.40403 \mathrm{KN} .
$$

The dimensionless parameters are given by

$$
\begin{gathered}
k_{f}=0.06366 ; \quad k_{1}=12.73239 ; \quad A_{s}=0.04899 ; \\
\alpha=0.00005 ; \quad b=0.006 ; \\
\omega_{1}=2.17656 ; \quad \omega_{2}=4.35312 .
\end{gathered}
$$

4.1. First Primary Resonance. In the following investigation, the normal continuous lines represent stable solutions and the broken lines represent the unstable solutions in all figures.

For the investigation of the system subjected to the principal resonance of the first mode in the presence of 2:1 internal resonance, the amplitude-frequency response curves along with their stability are obtained, as shown in Figures 3(a) and 3(b). The detuning parameter $\sigma$ which describes the nearness of $\omega$ to $\omega_{1}$ is taken as the control parameter. The amplitude-frequency response characteristics of $a_{2}$ are investigated, as the response of $a_{1}$ is obtained from the relationship between $a_{1}$ and $a_{2}$ shown in (23). Figure 3 shows that the response curves have both soft and hard characteristics. Therefore, the double-jumping phenomena are illustrated here. Furthermore, it is worth noting that there is no stable solution near the perfect first primary resonance at specific parameter combinations.

Amplitude-frequency response curves with the different amplitude of the external excitation, the viscoelastic coefficient, and the axial load are discussed in Figures 4 and 5. Figure 4(a) shows the amplitude-frequency response curves of the first primary resonance with three different external excitation amplitudes; that is to say, $b=0.004$, $b=0.006$, and $b=0.008$. Clearly, the height of the two resonance peaks and the bandwidth of the resonance are increasing with larger external excitation amplitude. Figure 4(b) shows the amplitude-frequency response curve of the first primary resonance corresponding to three different viscoelastic damping coefficients; that is, $\alpha=0.00001$, $\alpha=0.00005$, and $\alpha=0.0001$. The numerical results illustrate that the increasing viscoelastic damping decreases the amplitude of the resonance and shrinks the bandwidth of the resonance. Moreover, it is also seen that the viscoelastic damping has significant effect on the unstable regions. The resonance response may eventually degenerate into a single stable solution with the large viscoelastic damping.

When $P=143.40403 \mathrm{KN}, \varepsilon \sigma_{0}=0$ is obtained and the system is considered to be perfectly tuned. The detuning parameter $\sigma_{0}$ changes with the dimensionless parameter 


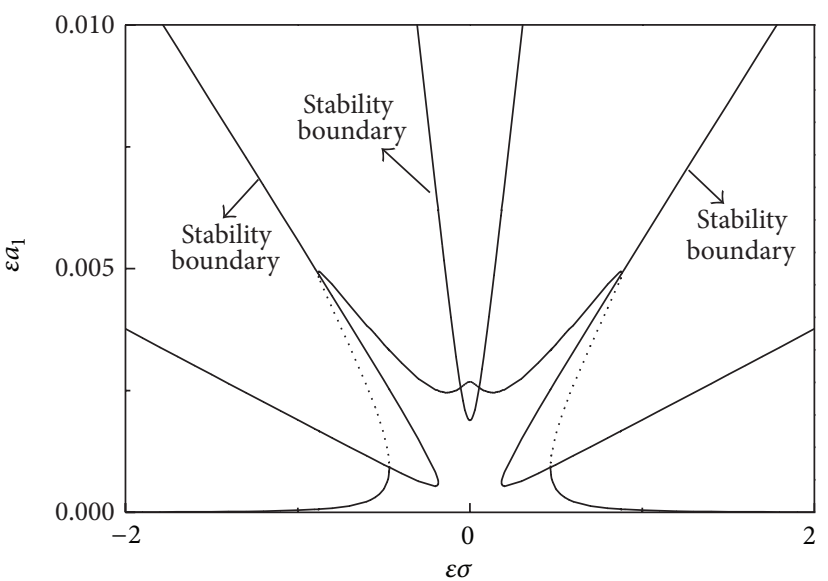

(a)

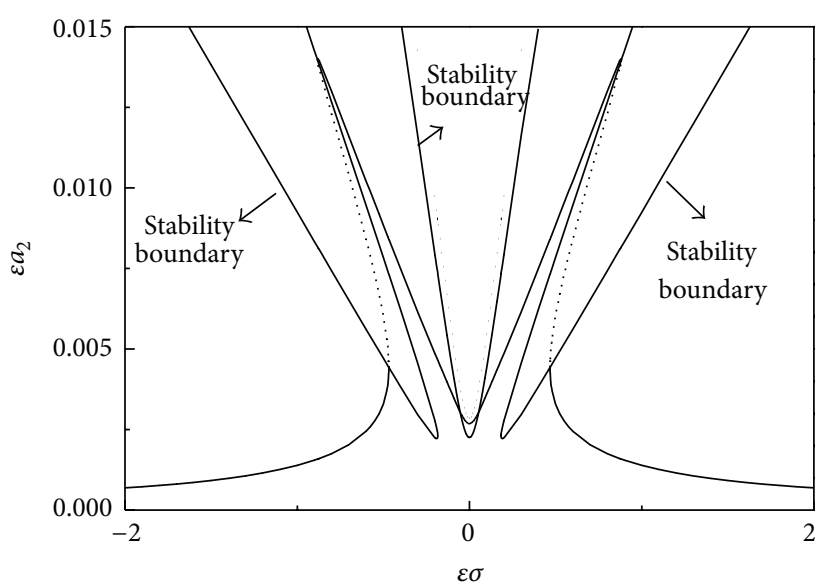

(b)

FIGURE 3: Amplitude-frequency response curves and stability boundaries of the system subjected to principal resonance of the first mode in the presence of $2: 1$ internal resonance: (a) $a_{1}$ mode; (b) $a_{2}$ mode.

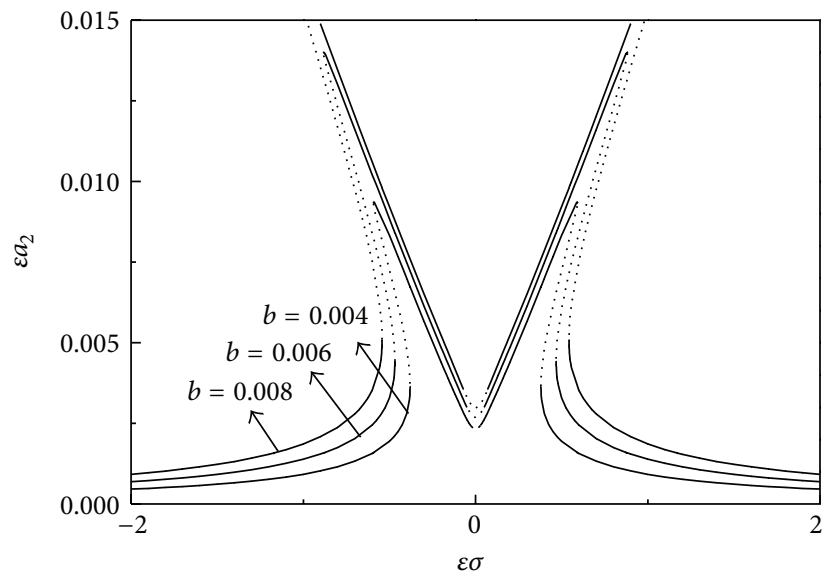

(a)

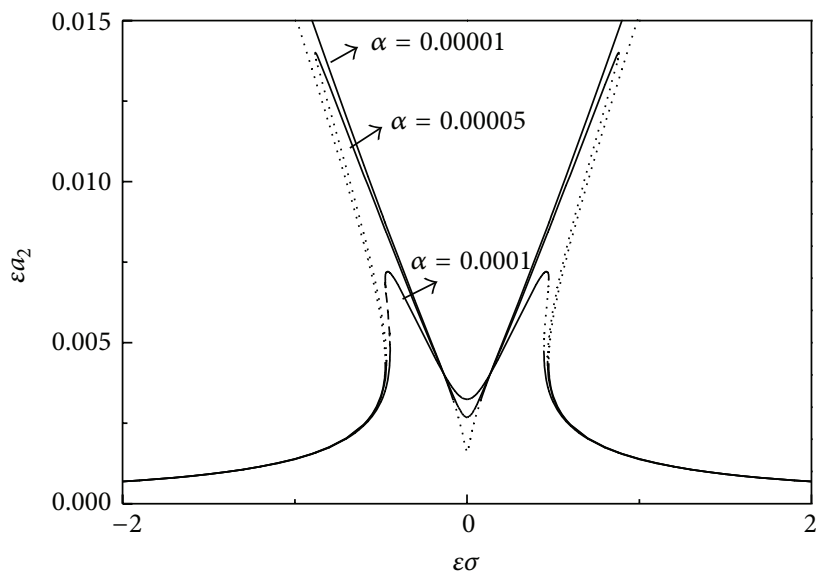

(b)

FIGURE 4: Amplitude-frequency response curves with different parameters: (a) different external excitation amplitudes and (b) different viscoelastic damping coefficients.

$k_{f}$ which is dependent on the axial load. If $\varepsilon \sigma_{0}=0.5$ and $\varepsilon \sigma_{0}=-0.5$ are taken into consideration, one derives $P=$ $181.25417 \mathrm{KN}$ and $P=116.38770 \mathrm{KN}$, respectively. Figure 5 shows the amplitude-frequency response curves of the first primary resonance with three different axial loads; that is to say, $P=143.40403 \mathrm{KN}, P=181.25417 \mathrm{KN}$, and $P=$ $116.38770 \mathrm{KN}$. As the axial load is increased, the height of the peak bending to the left is decreased and that of peak bending to the right is increased. Contrary results are obtained when decreasing the axial load.

4.2. Second Primary Resonance. In this section, the system subjected to the principal resonance of the second mode in the presence of $2: 1$ internal resonance will be discussed.

The amplitude-frequency response curves and stability boundaries are illustrated in Figure 6 . The detuning parameter $\sigma$ which describes the nearness of $\omega$ to $\omega_{2}$ is taken as the control parameter. Different from the previous first primary

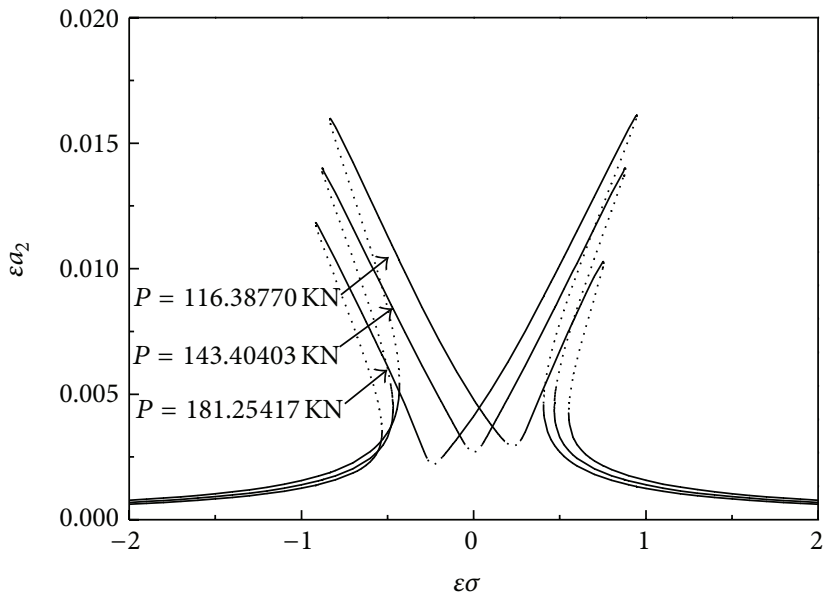

FIGURE 5: Amplitude-frequency response curves with different axial loads. 


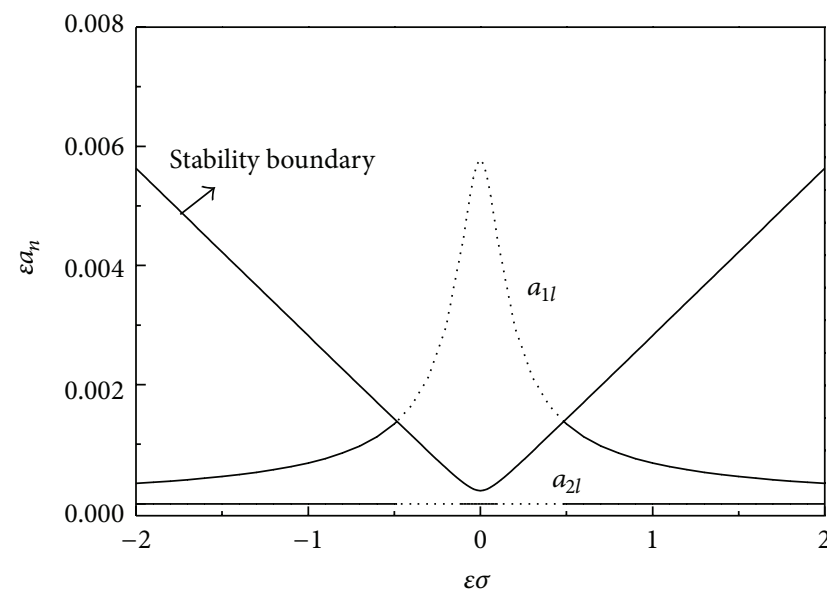

(a)

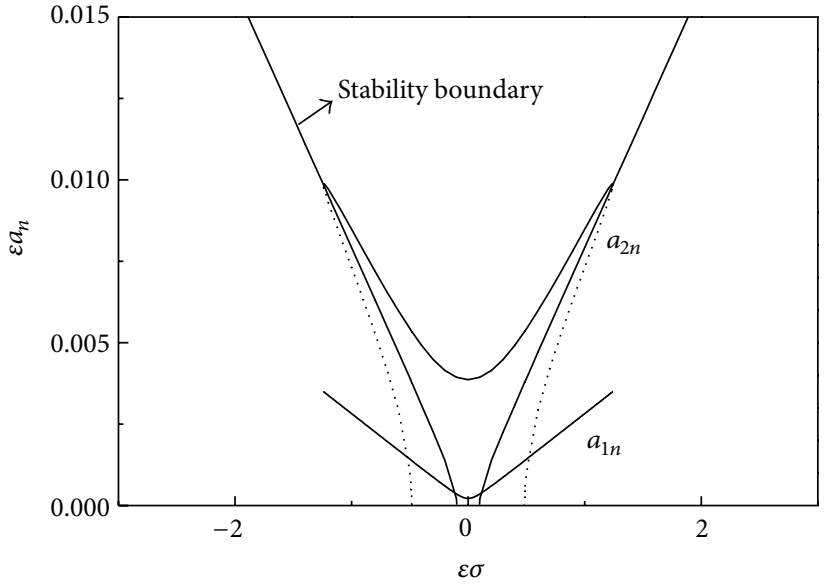

(b)

FIGURE 6: Amplitude-frequency response curves and stability boundaries of the system subjected to principal resonance of the second mode in the presence of 2:1 internal resonance: (a) local linear solution; (b) nonlinear solution.

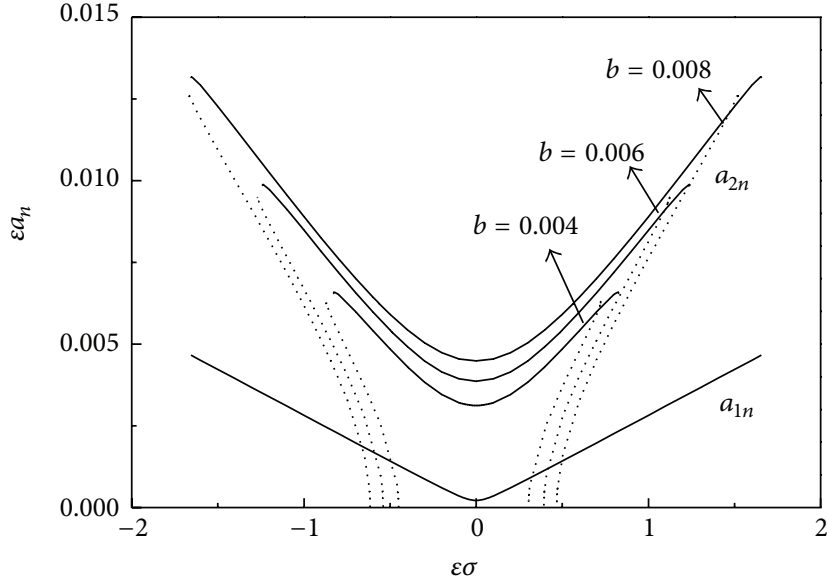

(a)

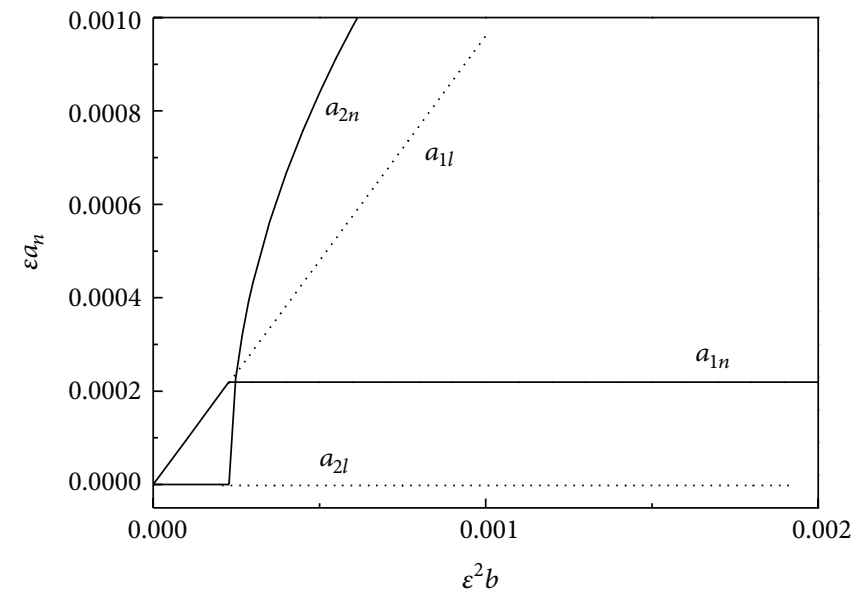

(b)

FIGURE 7: Effect of external excitation amplitude: (a) amplitude-frequency response curves with different amplitude of external excitation; (b) saturation phenomenon.

resonance, the response curves have a single mode and a coupled mode. Figure 6 shows the jump phenomenon between the single mode and the coupled mode. Nonlinear solution is activated by the principal resonance due to modal interaction. As local linear solution becomes unstable near the principal resonance, the nonlinear solution will be investigated in the following study. The amplitude-frequency response curves with the different amplitude of the external excitations, viscoelastic coefficients, and axial loads are obtained.

Figure 7 (a) shows the amplitude-frequency response curve of the second primary resonance with three different external excitation amplitudes; that is to say, $b=0.004$, $b=0.006$, and $b=0.008$. The modal amplitude response is depicted as function of the external excitation with the perfect primary resonance $(\varepsilon \sigma=0)$ and internal resonance $\left(\varepsilon \sigma_{0}=0\right)$. In Figure $7(\mathrm{a})$, as the external excitation amplitude is increased, the height of the peak of $a_{2}$ mode is increased and the bandwidth of resonance is expanded. On the other hand, the nonlinear stable solution of $a_{1}$ mode is not changed, which becomes saturated. As shown in Figure 7(b), the amplitude $a_{1}$ does not change eventually with the growing of excitation amplitude. There is an energy transfer from $a_{1}$ mode to $a_{2}$ mode. Namely, $a_{1}$ mode activates $a_{2}$ mode.

Figure 8(a) shows the amplitude-frequency response curve of the second primary resonance with three different viscoelastic damping coefficients; that is to say, $\alpha=0.00001$, $\alpha=0.00005, \alpha=0.0001$. Numerical results illustrate that the resonance peak of $a_{2}$ mode decreases with the increasing axial load near the principal resonance. On the other hand, the nonlinear stable solution of $a_{1}$ mode interestingly increases with the growing axial load. Figure 8 (b) shows the amplitudefrequency response curve of the second primary resonance with different axial loads; namely, $P=143.40403 \mathrm{KN}, P=$ 181.25417 KN, and $P=116.38770 \mathrm{KN}$ as the previous 


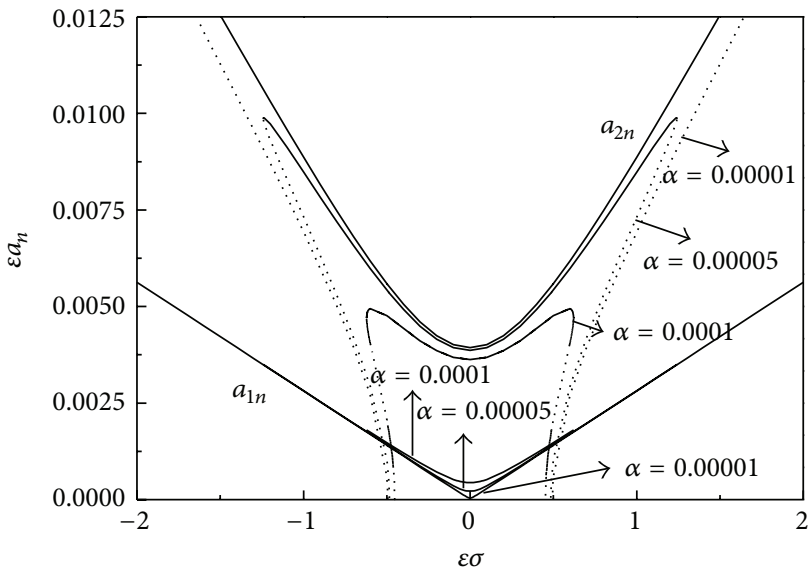

(a)

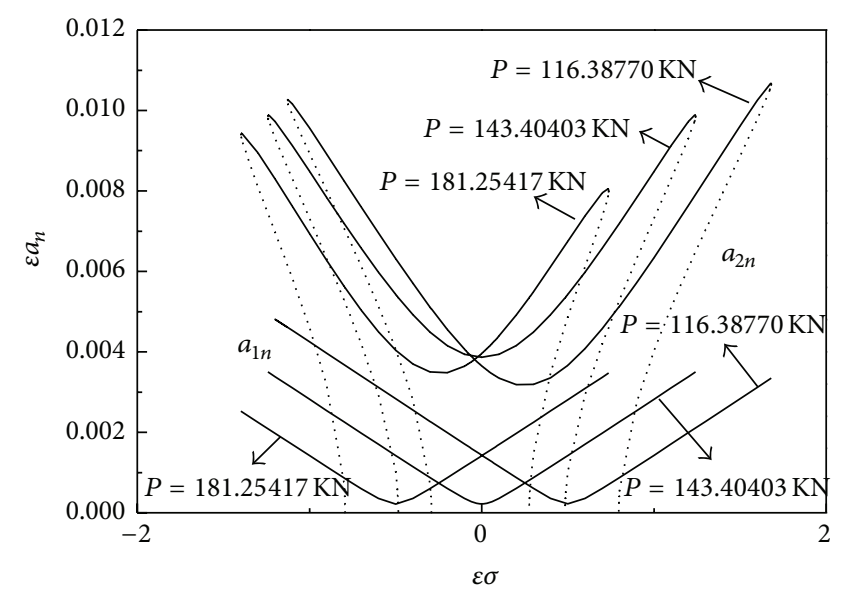

(b)

Figure 8: Amplitude-frequency response curves with different parameters: (a) different external viscoelastic damping coefficients; (b) different axial loads.

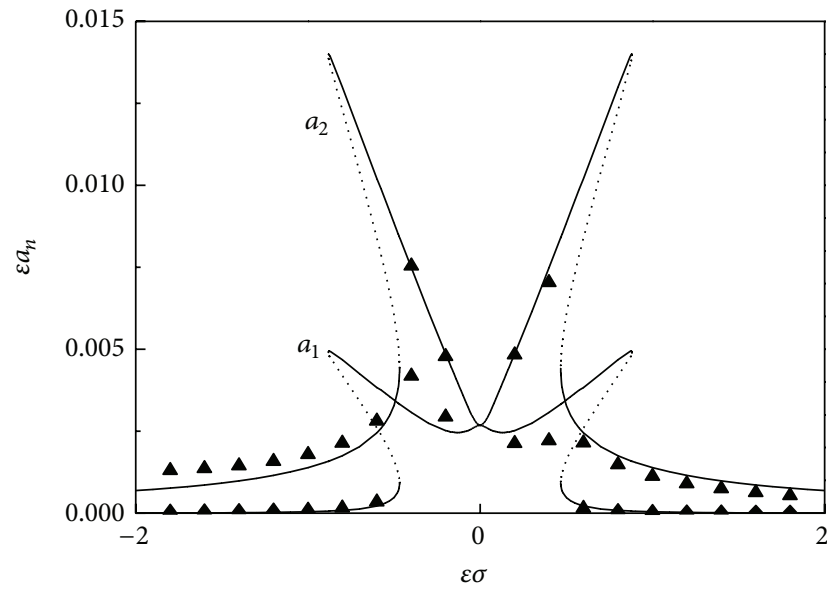

(a)

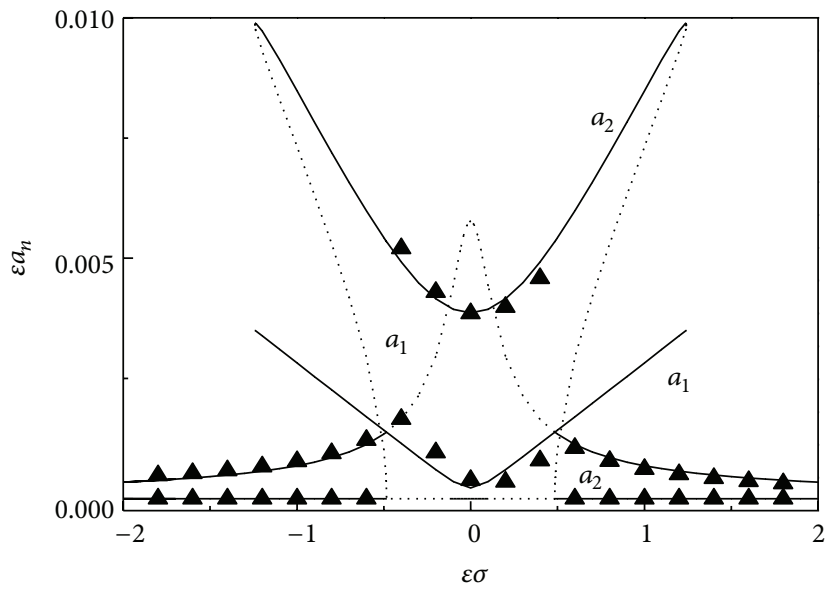

(b)

FIGURE 9: Comparison of amplitude-frequency response obtained by numerical method and approximate analytical method: (a) first primary resonance; (b) second primary resonance.

section. The numerical results in Figure 8(b) depict that the nonlinear resonance response curve moves to the left with the increasing axial load.

\section{Numerical Verification}

To verify the dynamic characteristics of the transverse weak forced vibration of the viscoelastic buckled beam, the fourthorder Runge-Kutta method is used to numerically calculate (11) which is defined as a two-degree-of-freedom linear system with small time-dependent nonlinear perturbations.

5.1. Comparison of Amplitude-Frequency Response. The values of the system parameters are still given by (38). The amplitude-frequency responses are obtained from periodic solutions with different excited frequencies. The normal continuous lines represent stable approximate analytical solutions, the broken lines represent the unstable approximate analytical solutions, and the triangle marks represent the numerical solutions.

Figure 9 shows that the results calculated by Runge-Kutta method and those obtained by the multiple scale method are in basic agreement. The double-jump phenomenon in the first primary resonance and the complex jump phenomenon between linear and nonlinear mode in the second primary resonance are verified. Under these parameters, the steadystate response near the perfect first primary resonance becomes unstable as the approximate analytical solutions shown in Figure 3.

5.2. Comparison of Time History Curve. The approximate analytical solutions of the time history response are obtained from (10), (12), (16), (20), (21), and (26). In order to verify the amplitude and the phase of the steady-state response, the four-order Runge-Kutta method is employed to obtain the time history response from (11). The normal continuous 


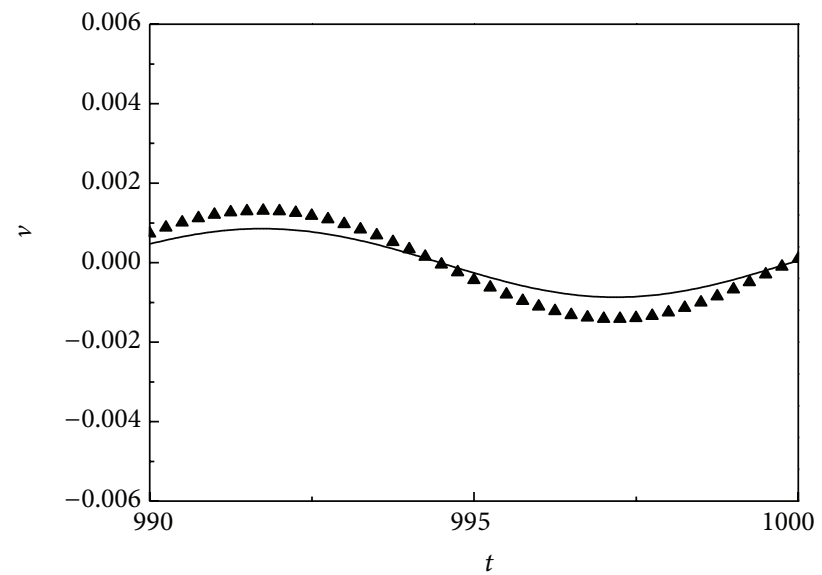

FIGURE 10: Time history response of quarter point under the first primary resonance at $\varepsilon \sigma=-1.6$.

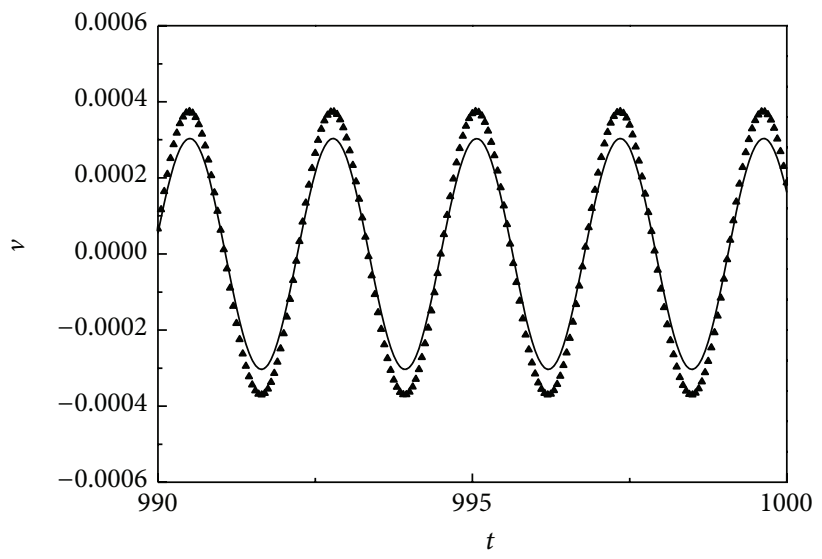

(a)

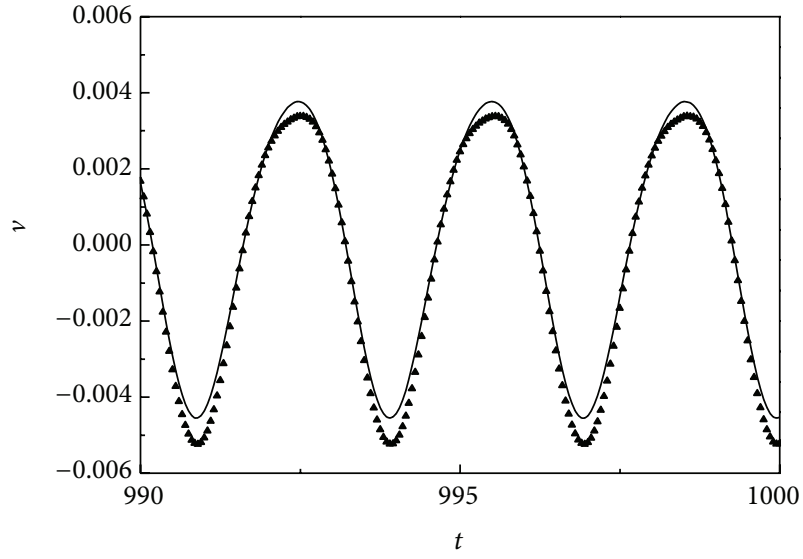

(b)

FIGURE 11: Time history response of quarter point under second primary resonance (a) at $\varepsilon \sigma=-0.2$; (b) at $\varepsilon \sigma=-1.6$.

lines represent stable approximate analytical solutions and the triangle marks represent the numerical solutions.

The dynamics of the quarter point of the beam is to be investigated. Due to the unstable regions near the perfect first primary resonance, $\varepsilon \sigma=-1.6$ is taken to verify the time history response far away from the perfect first primary resonance at first. Moreover, for the second primary resonance, $\varepsilon \sigma=-0.2$ and $\varepsilon \sigma=-1.6$ are taken to study the time history response near and far away from the perfect primary resonance, respectively. Figures 10 and 11 illustrate that the time history responses obtained by means of approximate analytical method are in basic agreement with numerical results in the case of the primary resonance and $2: 1$ internal resonance.

5.3. Verification for Dynamic Response of Higher Truncation Order. Equation (32) is defined as a four-degree-offreedom linear system with small time-dependent nonlinear perturbations. Calculating (32) by the fourth-order Runge-Kutta method yields the time history curve of the $q_{3}$ and $q_{4}$.

Figures 12 and 13 indicate the attenuation responses of $q_{3}$ and $q_{4}$ which are regarded as processes of free vibration.
The higher modes, which are not coupled with the first two modes by internal resonance under the harmonic excitations $f(x)=b \sin (2 \pi x)$ and $f(x)=b \sin (\pi x)$, have no effect on the steady-state response of the system.

5.4. Motion State Near the Perfect First Primary Resonance. As shown in Figures 3, 4, and 9, the periodic solutions are unstable in some regions near the perfect first primary resonance. These nonperiodic regions will be studied by constructing Poincaré maps for specific parameter combinations that are shown in Figure 14. The steady-state response of the quarter point of the beam is still selected to be discussed. Figures 14(a) and 14(b) show that quasiperiodic and period-doubling solutions are obtained with the variation of viscoelastic damping coefficient. For the enough small viscoelastic damping coefficient, there is also the possibility that the chaotic solution occurs, as shown in Figure 14(c). It is seen that the solutions are quasi-periodic under some excitation amplitudes and in other cases the period-doubling solutions may be observed in Figures 14(d) and 14(e). Enough large excitation amplitude will eventually lead to chaos as shown in Figure 14(f). 


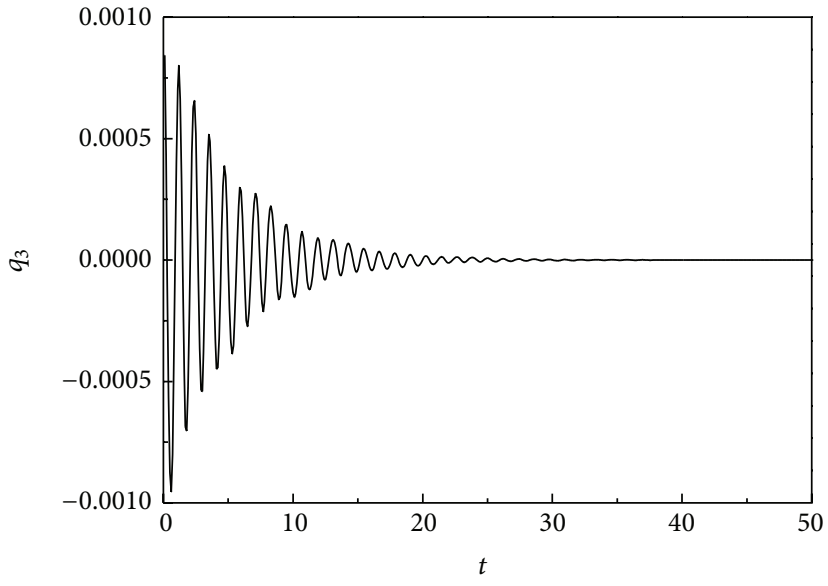

(a)

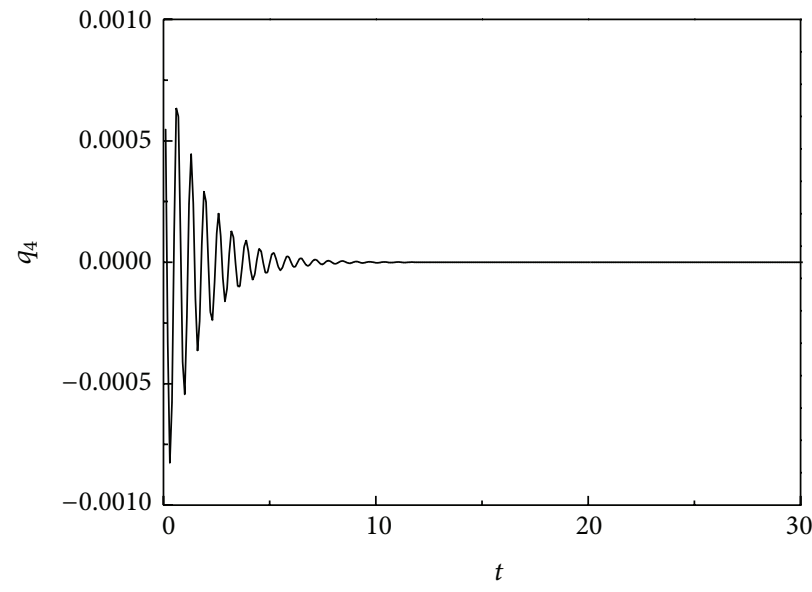

(b)

FIGURE 12: Time history response under first primary resonance of (a) $q_{3}$ mode; (b) $q_{4}$ mode.

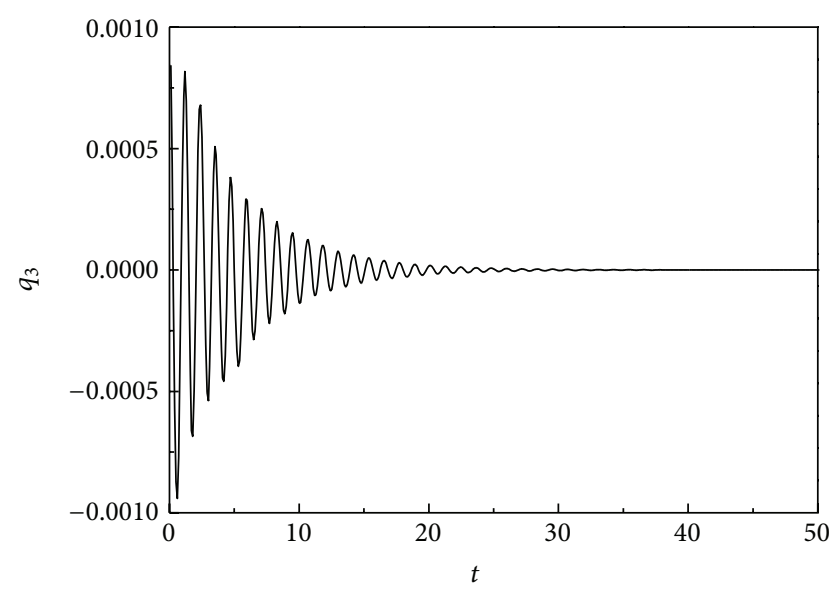

(a)

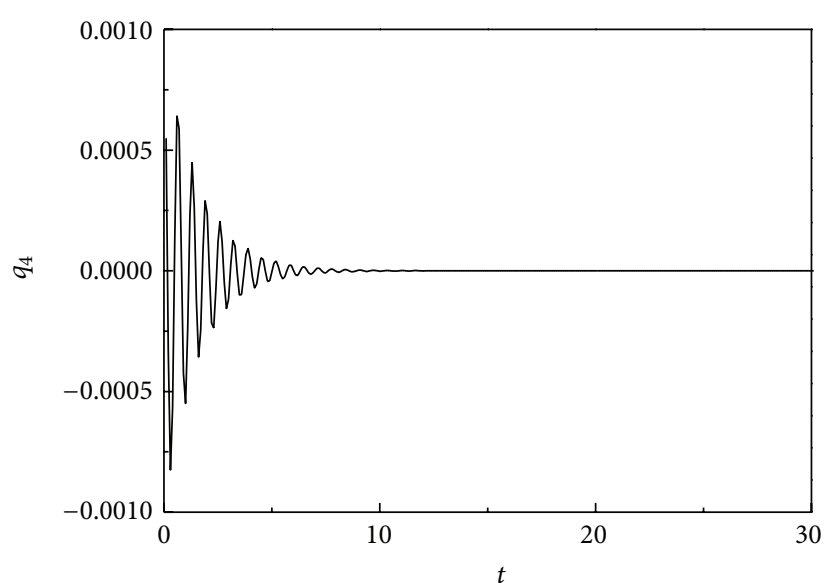

(b)

FIGURE 13: Time history response under second primary resonance of (a) $q_{3}$ mode; (b) $q_{4}$ mode.

\section{Conclusions}

The purpose of the present work is to study the influence of internal resonance on the dynamic response of the buckled beam with external distributed excitation. Due to the presence of quadratic and cubic nonlinearities in the equations of motion, analytical and numerical results indicate that the buckled beam displays a wealth of phenomena when subjected to dynamic loads. The following major conclusions are drawn from this study.

(1) Application of the Galerkin method truncates the governing equation into a two-degree-of-freedom system. The natural frequencies of the corresponding linear system are obtained. For specific axial load, two-to-one internal resonance in buckled beam may be activated.

(2) In the case of first primary resonance and 2:1 internal resonance, the amplitude-frequency response curves along with their stability are obtained. The doublejumping phenomenon with the change of detuning parameters can be detected. Response curves have both soft and hard characteristics.

(3) Under the conditions of second primary resonance and $2: 1$ internal resonance, unlike the first primary resonance, two possible steady-state solutions are recognized. The jump phenomenon in two possible modes and the saturation phenomenon are detected.

(4) The effects of external excitation amplitude, the viscoelastic damping, and the axial load on the resonance peak and the width of the resonance regions are discussed.

(5) Approximate analytical results are compared with the results obtained by Runge-Kutta numerical integrations. The agreement between the approximate analytical results and the numerical results is satisfactory. 


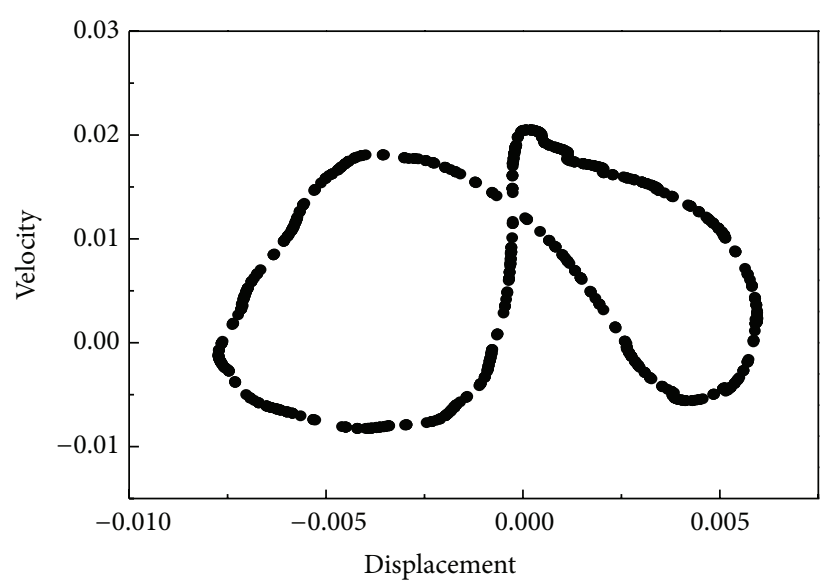

(a)

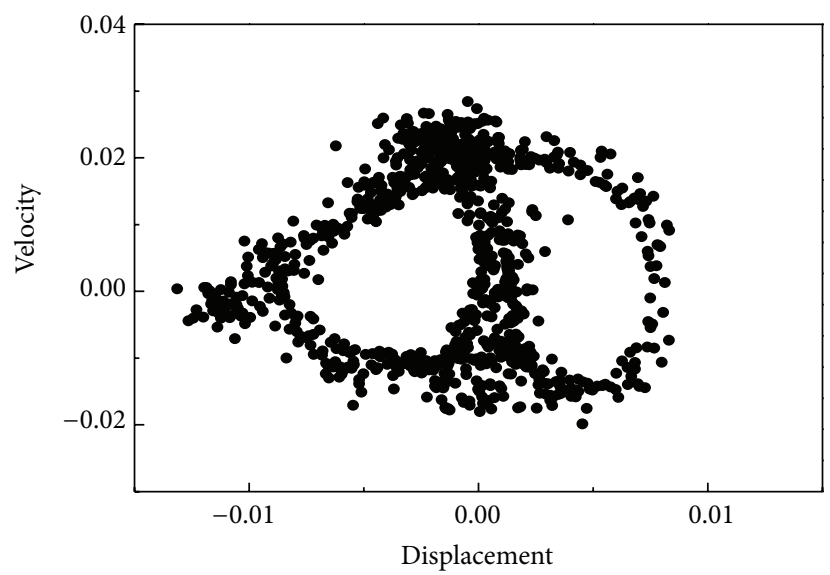

(c)

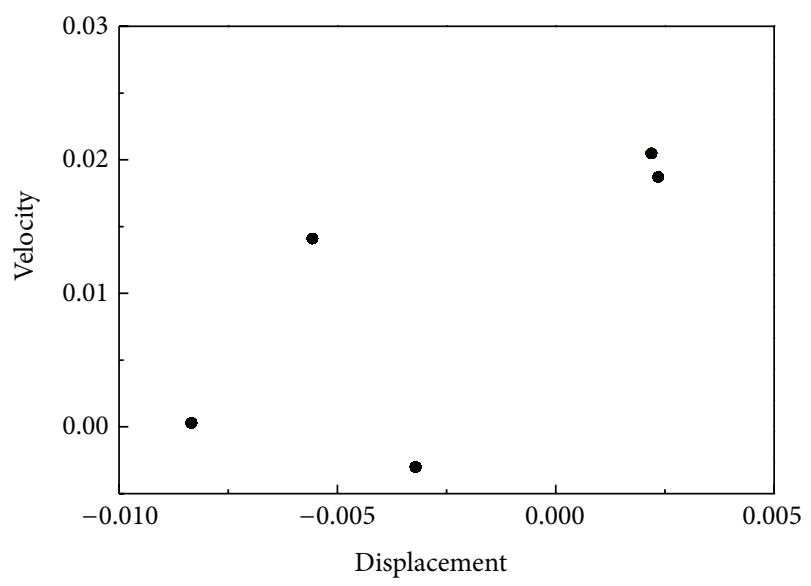

(e)

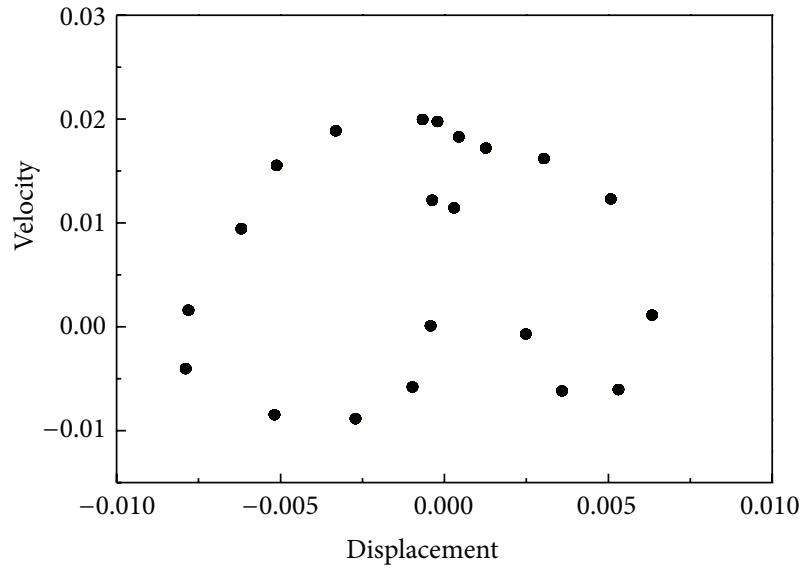

(b)

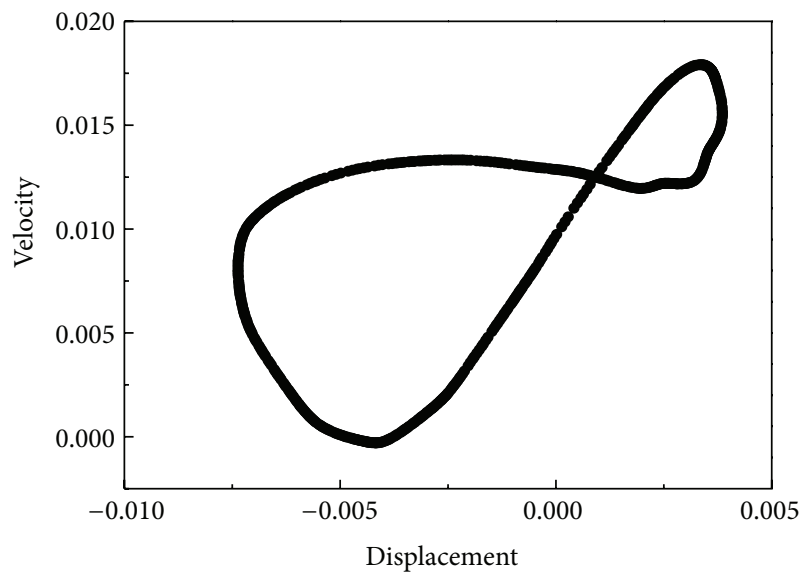

(d)

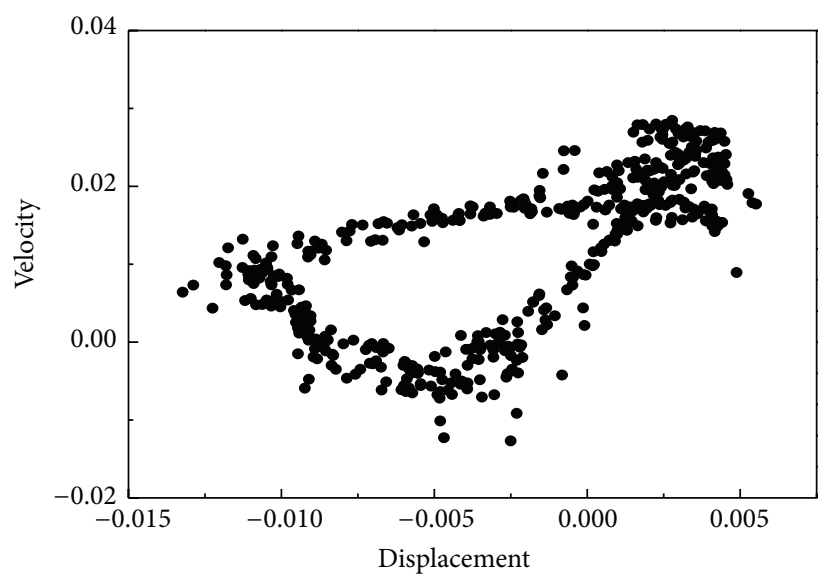

(f)

Figure 14: Poincaré maps for $=$ (a) $\alpha=0.000037, b=0.004$; (b) $\alpha=0.000032, b=0.004$; (c) $\alpha=0.00002, b=0.004$; (d) $\alpha=0.00005$, $b=0.006$; (e) $\alpha=0.00005, b=0.0084$; and (f) $\alpha=0.00005, b=0.01$.

(6) Nonperiodic region near the first primary resonance is discussed via numerical simulation. Poincaré maps are employed to demonstrate the transition from quasiperiodic and periodic doubling motions to chaos as the increase of the excitation amplitude and the decrease of viscoelastic damping. 


\section{Conflict of Interests}

The authors declare that there is no conflict of interests regarding the publication of this paper.

\section{Acknowledgments}

The authors gratefully acknowledge the support of the State Key Program of National Natural Science Foundation of China through Grant nos. 10932006 and 11232009, the National Natural Science Foundation of China through Grant no. 11372171, and the Innovation Program of Shanghai Municipal Education Commission through Grant no. 12YZ028.

\section{References}

[1] W. Y. Tseng and J. Dugundji, "Nonlinear vibrations of a buckled beam under harmonic excitation," Journal of Applied Mechanics, vol. 38, no. 2, pp. 467-476, 1971.

[2] G.-B. Min and J. G. Eisley, "Nonlinear vibrationsof buckled beams," Journal of Engineering for Industry, vol. 94, no. 2, pp. 637-646, 1972.

[3] A. H. Nayfeh, W. Lacarbonara, and C. M. Chin, "Nonlinear normal modes of buckled beams: three-to-one and one-to-one internal resonances," Nonlinear Dynamics, vol. 18, no. 3, pp. 253$273,1999$.

[4] C.-M. Chin and A. H. Nayfeh, "Three-to-one internal resonances in hinged-clamped beams," Nonlinear Dynamics, vol. 12, no. 2, pp. 129-154, 1997.

[5] W. Lacarbonara, A. H. Nayfeh, and W. Kreider, "Experimental validation of reduction methods for nonlinear vibrations of distributed-parameter systems: analysis of a buckled beam," Nonlinear Dynamics, vol. 17, no. 2, pp. 95-117, 1998.

[6] S. A. Emam and A. H. Nayfeh, "On the nonlinear dynamics of a buckled beam subjected to a primary-resonance excitation," Nonlinear Dynamics, vol. 35, no. 1, pp. 1-17, 2004.

[7] A. H. Nayfeh and B. Balachandran, "Modal interactions in dynamical and structural systems," Applied Mechanics Review, vol. 42, no. 11, pp. 175-201, 1989.

[8] C.-M. Chin and A. H. Nayfeh, "Three-to-one internal resonances in parametrically excited hinged-clamped beams," Nonlinear Dynamics, vol. 20, no. 2, pp. 131-158, 1999.

[9] A. A. Afaneh and R. A. Ibrahim, "Nonlinear response of an initially buckled beam with 1:1 internal resonance to sinusoidal excitation," Nonlinear Dynamics, vol. 4, no. 6, pp. 547-571, 1993.

[10] C. Chin, A. H. Nayfeh, and W. Lacarbonara, "Two-to-one internal resonances in parametrically excited buckled beams," in Proceedings of the 38th Structures, Structural Dynamics, and Materials, no. 97-1081, AiAA, Kissmmee, Fla, USA, 1997.

[11] S. P. Machado and C. M. Saravia, "Shear-deformable thinwalled composite Beams in internal and external resonance," Composite Structures, vol. 97, pp. 30-39, 2013.

[12] S. A. Emam and A. H. Nayfeh, "Non-linear response of buckled beams to 1:1 and 3:1 internal resonances," International Journal of Non-Linear Mechanics, vol. 52, pp. 12-25, 2013.

[13] C. W. de Silva, Vibration Damping, Control, and Design, CRC Press, Taylor \& Francis Group, 2007.

[14] W.-R. Chen, "Bending vibration of axially loaded Timoshenko beams with locally distributed Kelvin-Voigt damping," Journal of Sound and Vibration, vol. 330, no. 13, pp. 3040-3056, 2011.
[15] L. Galuppi and G. Royer-Carfagni, "Buckling of three-layered composite beams with viscoelastic interaction," Composite Structures, vol. 107, pp. 512-521, 2014.

[16] A. H. Nayfeh, D. T. Mook, and L. R. Marshall, "Nonlinear coupling of pitch and roll modes in ship motions," Journal of Hydronautics, vol. 7, no. 4, pp. 145-152, 1973.

[17] A. H. Nayfeh and D. T. Mook, Nonlinear Oscillations, Wiley Interscience, New York, NY, USA, 1979.

[18] A. Alasty and R. Shabani, "Chaotic motions and fractal basin boundaries in spring-pendulum system," Nonlinear Analysis: Real World Applications, vol. 7, no. 1, pp. 81-95, 2006.

[19] L. Q. Chen, Y. L. Zhang, G. C. Zhang, and H. Ding, "Evolution of the double-jumping in pipes conveying fluid flowing at the supercritical speed," International Journal of Non-Linear Mechanics, vol. 58, pp. 11-21, 2014. 


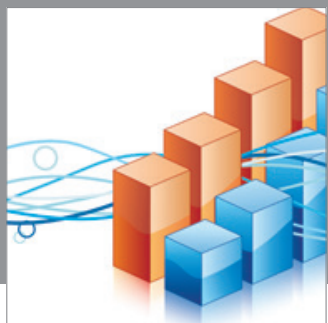

Advances in

Operations Research

mansans

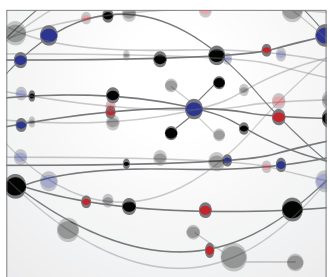

The Scientific World Journal
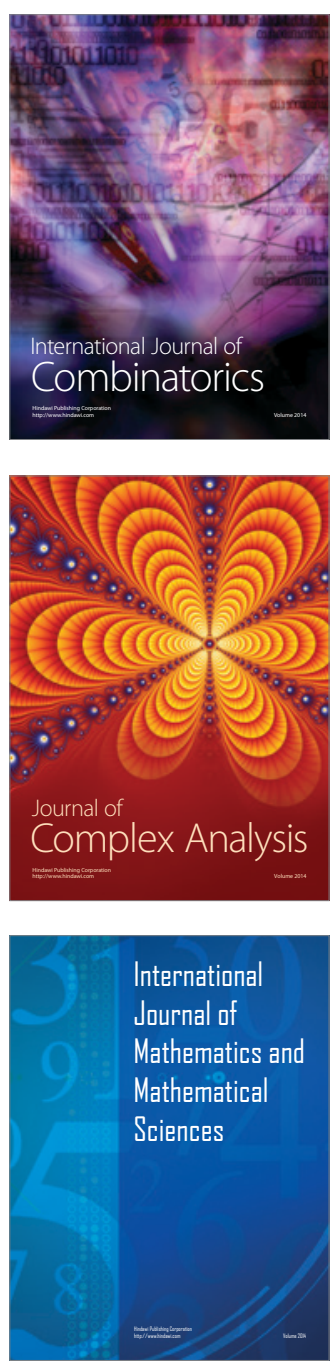
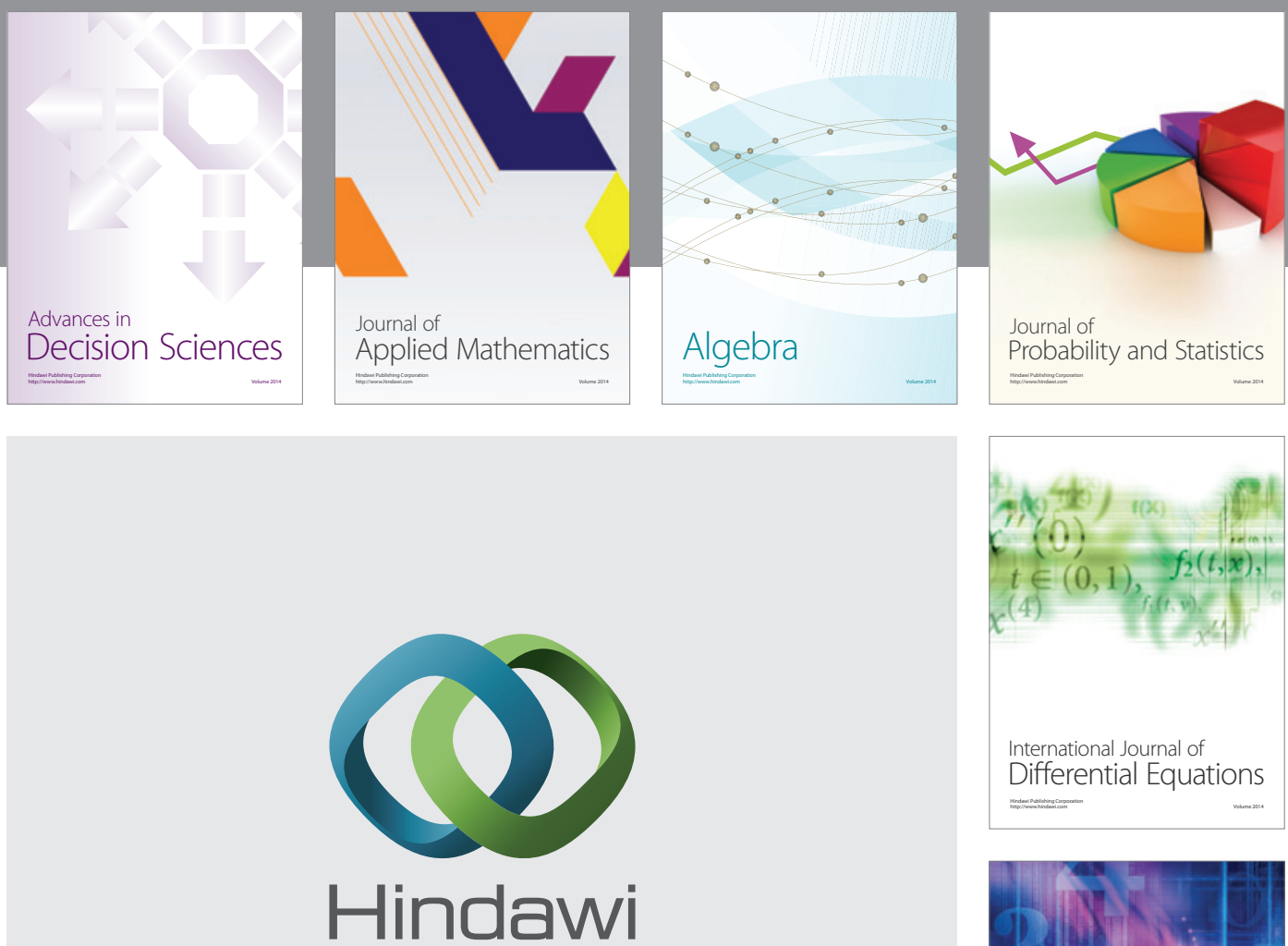

Submit your manuscripts at http://www.hindawi.com
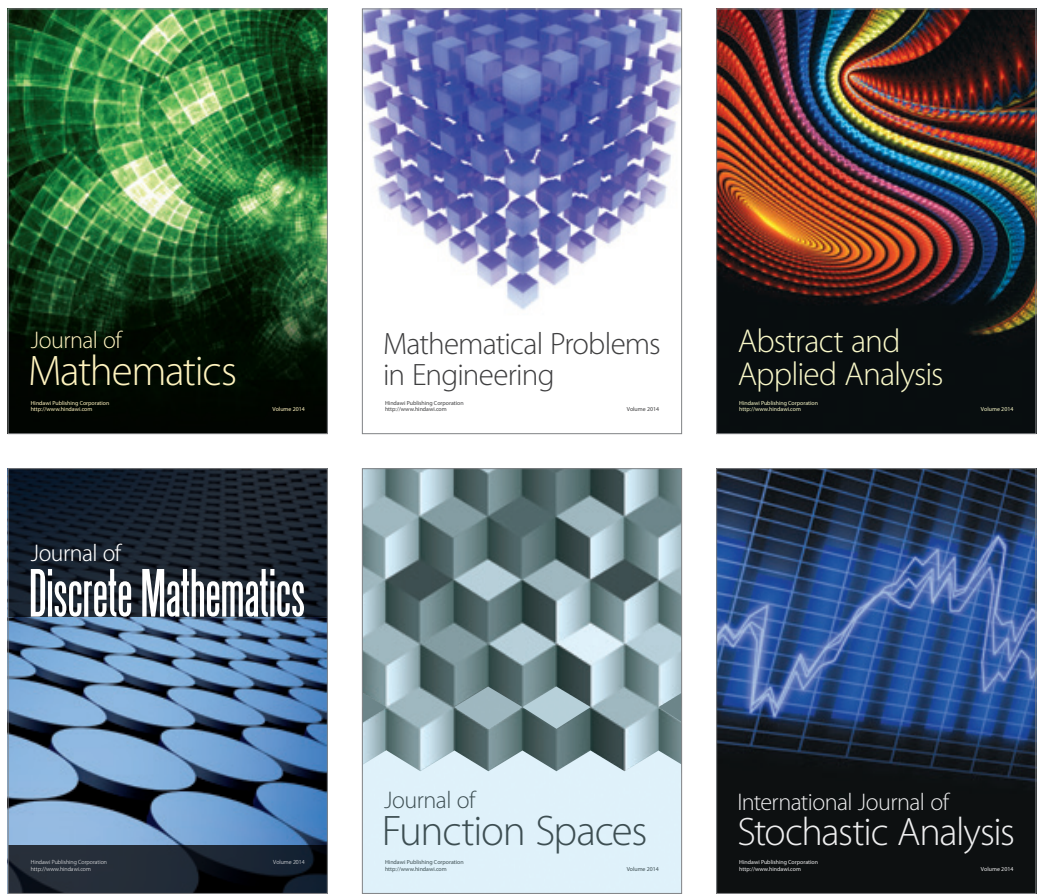

Journal of

Function Spaces

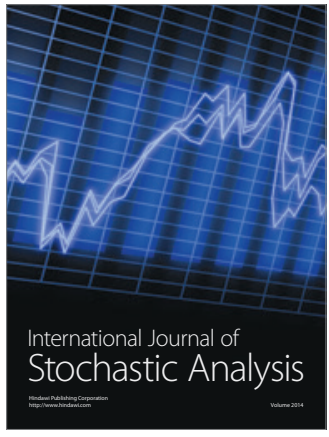

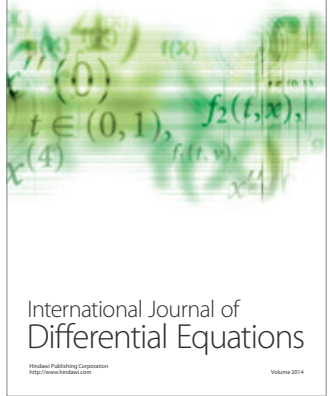
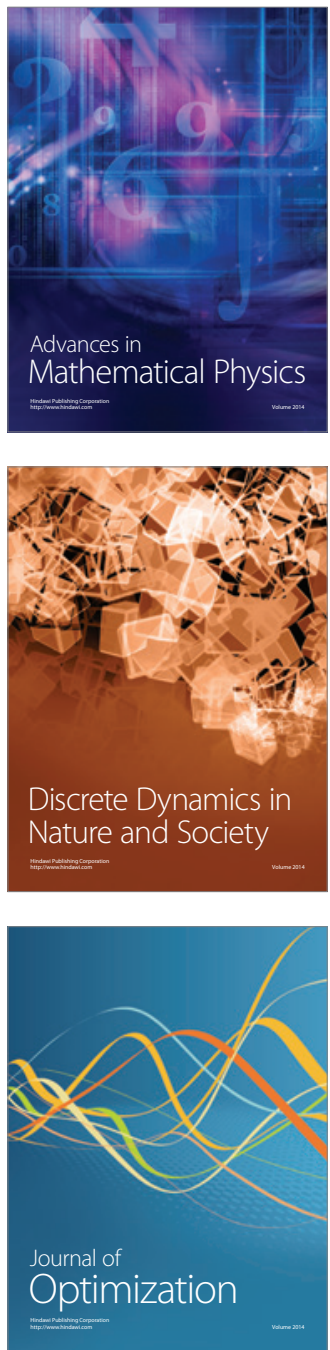